\title{
Dissection of MAPK signaling specificity through protein engineering in a developmental context
}

\author{
Diego L. Wengier ${ }^{1,2^{*}}$, Gregory R. Lampard ${ }^{1}$ and Dominique C. Bergmann ${ }^{1,3^{*}}$
}

\begin{abstract}
Background: Mitogen-activated protein kinases (MAPK) signaling affects many processes, some of which have different outcomes in the same cell. In Arabidopsis, activation of a MAPK cascade consisting of YODA, MKK4/5 and MPK3/6 inhibits early stages of stomatal developmental, but the ability to halt stomatal progression is lost at the later stage when guard mother cells (GMCs) transition to guard cells (GCs). Rather than downregulating cascade components, stomatal precursors must have a mechanism to prevent late stage inhibition because the same MKKs and MPKs mediate other physiological responses.

Results: We artificially activated the MAPK cascade using MKK7, another MKK that can modulate stomatal development, and found that inhibition of stomatal development is still possible in GMCs. This suggests that MKK4/5, but not MKK7, are specifically prevented from inhibiting stomatal development. To identify regions of MKKs responsible for cell-type specific regulation, we used a domain swap approach with MKK 7 and a battery of in vitro and in vivo kinase assays. We found that N-terminal regions of MKK5 and MKK7 establish specific signal-to-output connections like they do in other organisms, but they do so in combination with previously undescribed modules in the C-terminus. One of these modules encoding the GMC-specific regulation of MKK5, when swapped with sequences from the equivalent region of MKK7, allows MKK5 to mediate robust inhibition of late stomatal development.
\end{abstract}

Conclusions: Because MKK structure is conserved across species, the identification of new MKK specificity modules and signaling rules furthers our understanding of how eukaryotes create specificity in complex biological systems.

Keywords: MAPK specificity, Signal transduction, Network rewiring, Stomatal development

\section{Background}

MAPKs have strategic roles in signal processing, in mediating stress responses, and in guiding cell fate transitions and development [1-4]. MAPK networks consist of a three-tiered cascade whose kinases-MAPK kinase kinase or MKKK, MAPK kinase (Mapk/Erk kinases or MEK in animals, MKK in Arabidopsis) and MAPK (MPK in Arabidopsis) sequentially phosphorylate and activate each other upon signal perception. Downstream effectors may respond to MPK-mediated phosphorylation by changes in protein activity, localization or stability, and many of these alterations ultimately alter transcriptional programs. The MKK level is a bottleneck in many species.

\footnotetext{
* Correspondence: dwengier@dna.uba.ar; dbergmann@stanford.edu

${ }^{1}$ Howard Hughes Medical Institute, Chevy Chase, USA

Full list of author information is available at the end of the article
}

In humans, at least 25 MKKKs activate the 7 MEKs, which lie upstream of 14 MAPKs [1]. In Arabidopsis thaliana, more than 60 MKKKs are predicted upstream of 10 MKKs and 23 MPKs [5]. Evidence exists that MKKs can activate more than one MPK, and a given MPK may have more than one upstream MKK [6]. Intuitively, this arrangement could facilitate signal integration, as multiple signals could converge on a single effector. The use of common components, however, could also lead to erroneous crossactivation.

When expressing multiple MAPK network components and responding to multiple sources of information, how do cells generate an appropriate output to a particular signal? One strategy is to make downstream effectors available in only certain cells [7] or under certain conditions [8-10]. Alternatively, signaling networks can be insulated through the use of scaffolds, subcellular partitioning 
of signaling complexes, and by varying signal amplitude or duration [1, 5, 11-14]. Some MAPK network components encode regions that allow them to establish these connections or localizations. For instance, the animal MEK1/2 uses its proline-rich sequence (PRS) to bind the scaffolds Kinase Suppressor of Ras (KSR) and Mek Partner 1 (MP1). Binding to MP1, in particular, mediates endosomal localization [15-17]. Several human MEKs and all Arabidopsis MKKs lack a PRS, however, leaving questions about how these smaller MKKs are correctly assembled into restricted MAPK networks.

In Arabidopsis, MAPK signaling has fundamental roles in development and organogenesis $[18,19]$ including in the formation of stomata, the structures in the epidermis of plants that regulate gas exchange [20,21]. Because of this central role of MAPK signaling in development, studying how this cascade regulates particular cell fate decisions with traditional genetic approaches is quite challenging. New strategies that dissect specific responses in selected tissues must be designed to replace analysis of mutants with such complex, interlinked phenotypes. Such strategies include the use of inducible or cell type-specific promoters and constitutively active (CA) or dominant negative variants of MAPK components. Although these variants do not exist in nature, they mimic situations of cell type-specific regulation of the MAPK cascade. Previously, we used a systematic study of cell stage-specific responses to MAPK activation and revealed that stomatal precursors have mechanisms to limit certain cellular outputs and generate MKK-specific responses [22, 23]. Only four of the 10 MKKs -MKK4, MKK5, MKK7 and MKK9have any capacity to influence stomatal development during lineage initiation, guard mother cell (GMC) commitment and/or guard cell (GC) formation (for simplicity, only MKK5 and MKK7 are shown in Fig. 1). Expression of any of these CA-MKKs strongly inhibits stomatal formation in early development (Fig. 1a, b and c). At the last stage of development, however, MKK4 and MKK5 lose their ability to inhibit stomatal formation (Fig. 1d and e). MKK7 and MKK9 activation, in contrast, results in stomatal clustering (Fig. 1d and $\mathrm{f}$ ). Loss of function studies with $M K K 4$ and $M K K 5$ indicate that these kinases are

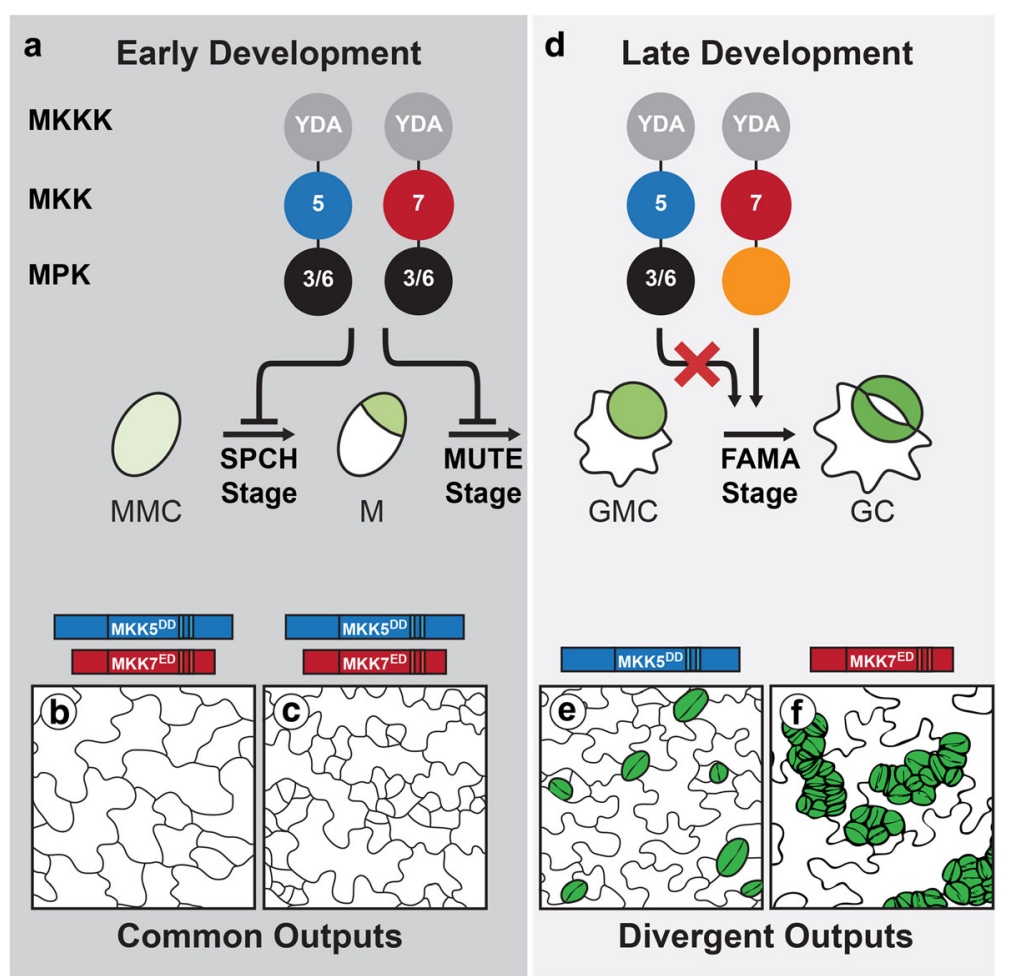

Fig. 1 Schematic of stomatal lineage, indicating stages where MKK activation leads to similar and divergent outputs (based on [22]). Diagrams of MAPK signaling cascades, with each colored circle representing a different kinase level; circles are labeled with relevant kinase number, with orange circle representing MPK of unknown identity. Constitutive activation of YDA (MKKK), MKK5 or MKK7 inhibits stomatal lineage initiation (a, SPCH and MUTE stages). Late in the lineage (d, FAMA stage) YDA and MKK7, but not MKK5, activation leads to stomatal proliferation via an unidentified MPK. b, c, e, $\mathbf{f}$ Tracings of phenotypes resulting from activation of kinases. In (b-c), constitutively active MKK5 (MKK5 ${ }^{\mathrm{DD}}$ ) or MKK7 (MKK $7^{\mathrm{ED}}$ ) inhibit initiation (division of meristemoid mother cell $(M M C)$ into meristemoid $(M)$ ) and lineage progression (conversion of $M$ into guard mother cell $(G M C)$ ). In (e-f), MKK5 ${ }^{\mathrm{DD}}$ has no effect (e, WT numbers and distribution of stomata in green), but MKK $7^{\mathrm{ED}}$ induces guard cell (GC) overproliferation and clustering (f). Stages are referred to as SPCH, MUTE and FAMA after the promoters to drive expression of MAPK network components [22]. YDA, YODA; 5, MKK5; 7, MKK7; 3/6, MPK3 and MPK6 
endogenously required to limit stomatal production [21], but the MKK7/9 significance is unclear as $m k k 9$ single mutants do not affect stomata and in the recent report of true loss of function mutations in $m k k 7 \mathrm{mu}-$ tants no stomata phenotype was described [19]. MKK4/ 5 's endogenous role in limiting stomatal production in early stages, but not in late stomatal lineage cells, provides an excellent test case for examining how cell identity may interface with signaling response.

In this study, we created a quantitative phenotypic analysis pipeline that revealed a previously underappreciated capacity for late stage stomatal lineage cells to be inhibited by MAPK signaling. Then, taking advantage of divergent responses to constitutively activated MKK5 and MKK7, we implemented a protein engineering approach to identify structural domains in MKK5 that are responsible for its stage-specific behaviors. We found that MKK N-terminal regions establish specific signalto-output connections, much like they do in other organisms [24], but this requires coordination with previously unexplored regions in the C-terminus. We also found that a minimal domain in the $\mathrm{C}$-terminus encodes the basis for MKK-specific regulation. The location of specificity modules within the plant proteins corresponds to regions in which their human homologues display high sequence diversity, suggesting that these regions may contribute to specificity in many situations. Given the global conservation of MAPK signaling, our findings in the complex multicellular context of plant development may offer insights into general mechanisms of signaling specificity in complex biological systems.

\section{Results}

Stomatal development is inhibited by MAPK activation at the FAMA stage

To carefully define the range of phenotypes in our system, we re-analyzed the inhibitory effect of CA-MKK expression in FAMA stage cells (Fig. 1d) using more sensitive and quantitative measurements than in our previous studies [22, 23]. For simplicity, we selected one representative MKK from each MKK4/5 and MKK7/9 pair as previous studies showed that MKK4 mirrors MKK5 activity, and MKK7 mirrors MKK9 activity, in every stage of stomatal development $[22,23]$. Because MKKs were to be analyzed in planta, we selected MKK5 and MKK7, both of which can be easily detected as YFP fusion proteins, thereby providing a control for expression. FAMA promoter (FAMApro) was used to drive the expression of constitutively active MKKs which are made dominantly active by replacing the regulatory $\mathrm{S} / \mathrm{T}$ residues of the activation loop with phosphomimetic $\mathrm{D} / \mathrm{E}$ residues $\left(\mathrm{MKK} 5^{\mathrm{DD}}=\right.$ $\mathrm{MKK}^{\mathrm{T} 215 \mathrm{D}, \mathrm{S221D}}$ and MKK $\left.7^{\mathrm{ED}}=\mathrm{MKK}^{\mathrm{S} 193 \mathrm{E}, \mathrm{S199D}}\right)[22,25]$. To be able to observe all epidermal phenotypes produced by different MKK expression levels, such as inhibition of stomatal development and stomatal clustering, phenotypes were quantified in cotyledons of independent primary transformants (T1 s in Table 1, Fig. 2a). We paid special attention to evidence of seedling lethality, a typical result of inhibition of stomatal development. Previously, expression of $M K K 7^{\mathrm{ED}}$ was shown to lead to stomatal hyperproliferation [22], and we could confirm that result: $54 \% \mathrm{~T} 1 \mathrm{~s}$ showed large stomatal clusters (Fig. 2a and b, Table 1); however, 26\% were WT (most of which showed no YFP signal) and $20 \%$ had stomatal precursors that failed to complete their development into GCs (Fig. 2a and b, Inhibited). This third class died as seedlings. Among MKK $5^{\mathrm{DD}}$-YFP transformants, there were no seedling lethals: $76 \% \mathrm{~T} 1 \mathrm{~s}$ had a phenotype indistinguishable from controls (Fig. 2b, Table 1) and 24\% exhibited one to three small clusters (2-3 stomata in contact) per 0 . $32 \mathrm{~mm}^{2}$. Among control seedlings grown in parallel, $8 \%$ exhibited similar small clusters (Table 1). Phenotype distributions for MKK5 ${ }^{\mathrm{DD}}$-YFP and $\mathrm{MKK} 7^{\mathrm{ED}}$-YFP were statistically different in Chi-squared test of independence $(p<0.05)$.

These results indicate that MAPK activation through $M K K 7^{\mathrm{ED}}$, besides driving stomatal clustering, can also lead to inhibition of stomatal development at the FAMA stage. The failure of $M K K 5^{\mathrm{DD}}$ to inhibit this stage transition is puzzling, since MKK5 is the endogenous kinase, whereas MKK7 is not normally expressed in FAMA stage cells [26]. Moreover, MKK $5^{\mathrm{DD}}$ is an effective inhibitor of earlier stages [22] and MKK4MKK5RNAi lines show excess mature GCs [21]. Also, when compared to MKK $7^{\mathrm{ED}}$, MKK5 exhibits stronger interactions with MPK3/6 in Y2H (Additional file 1: Figure S1) and stronger kinase activity in vitro (Additional file 2: Figure S2). MKK5, therefore appears to be subject to an additional level of in vivo regulation that blocks its inhibitory effect, while MKK7 seems to escape this regulation. We reasoned that structural differences between MKK5 and MKK7 could be probed to define the nature and the source of this differential regulation.

\section{Predicted tertiary structures of MKK5 and MKK7 suggest sources of MKK identity and specificity}

We reasoned that the domains most likely to confer the FAMA-stage differential responses would be surface exposed (thus available for interactions with partners) and would exhibit the greatest structural and sequence divergence among MKKs. To facilitate the identification of such regions, we modeled plant MKK folds based on the X-ray crystal structures of human orthologs MEK1 and MEK2 using I-Tasser [27] (Fig. 3) and used structural information from several other mammalian kinases [28-31] to identify conserved features.

Structural alignment of MKK5 and MKK7 to mammalian kinases confirmed the conservation of the 
Table 1 Quantification of in vivo and in vitro phenotypes conferred by chimeras

\begin{tabular}{|c|c|c|c|c|c|c|c|c|c|c|c|c|}
\hline \multirow[t]{3}{*}{ Name } & \multicolumn{4}{|c|}{$\begin{array}{l}\text { Kinase Activity (normalized } \\
\text { to } M K K 5^{D D} \text { ) }\end{array}$} & \multicolumn{2}{|c|}{$\begin{array}{l}\text { SPCH stage inhibition } \\
\text { of linage initiation }\end{array}$} & \multicolumn{6}{|c|}{$\begin{array}{l}\text { FAMA stage phenotypes (as percentage } \\
\text { of total } T 1 \text { seedlings) }\end{array}$} \\
\hline & \multicolumn{2}{|c|}{ KI-MPK3 } & \multicolumn{2}{|l|}{ KI-MPK6 } & \multirow[t]{2}{*}{$n$} & \multirow[t]{2}{*}{$\%$} & \multirow[t]{2}{*}{$n$} & \multirow[t]{2}{*}{ Inhibited } & \multirow[t]{2}{*}{ WT } & \multicolumn{3}{|c|}{ GC clusters } \\
\hline & AVG & SE & AVG & SE & & & & & & Small & Large & Total \\
\hline YFP control & & & & & & & 24 & 0.00 & 91.67 & 8.33 & 0.00 & 8.33 \\
\hline $\mathrm{MKK} 5^{\mathrm{DD}}$ & 34.485 & 9.052 & $38.259^{*}$ & 2.308 & 69 & 78.26 & 25 & 0.00 & 76.00 & 24.00 & 0.00 & 24.00 \\
\hline$M K K 7^{E D}$ & 6.836 & 4.808 & 10.867 & 5.726 & 93 & 100.00 & 50 & 20.00 & 26.00 & 0.00 & 54.00 & 54.00 \\
\hline N7-MKK5 DD & 5.500 & 1.894 & 21.448 & 9.176 & 75 & 26.67 & 125 & 8.80 & 32.00 & 48.80 & 10.40 & 59.20 \\
\hline N5-MKK7 ED & 15.889 & 7.052 & $35.235^{*}$ & 1.457 & 22 & 86.36 & 47 & 93.62 & 6.38 & 0.00 & 0.00 & 0.00 \\
\hline $\mathrm{MKK} 5^{\mathrm{DD}}-\mathrm{C} 7$ & 13.888 & 9.753 & $25.666^{*}$ & 2.530 & 47 & 87.23 & 44 & 65.91 & 29.55 & 4.55 & 0.00 & 4.55 \\
\hline MKK5 $5^{\mathrm{DD}}-7 \mathrm{~A}$ & 45.980 & 22.674 & 26.287 & 4.947 & 114 & 12.28 & 98 & 0.00 & 97.96 & 2.04 & 0.00 & 2.04 \\
\hline$M K K 5^{D D_{-7 B}}$ & 46.746 & 22.671 & $38.718^{*}$ & 0.750 & 20 & 10.00 & 70 & 50.00 & 50.00 & 0.00 & 0.00 & 0.00 \\
\hline$M K K 7^{E D}-C 5$ & 3.877 & 2.091 & 0.728 & 0.148 & 43 & 13.95 & 19 & 0.00 & 78.95 & 21.05 & 0.00 & 21.05 \\
\hline MKK7 ${ }^{\mathrm{ED}}$-CDR5 & 2.451 & 1.242 & $1.303^{*}$ & 0.312 & 89 & 98.81 & 30 & 96.67 & 3.33 & 0.00 & 0.00 & 0.00 \\
\hline MKK7 ${ }^{\text {ED }}$-CPR5 & 15.844 & 11.444 & 5.904 & 2.422 & 105 & 0.00 & 36 & 2.78 & 91.67 & 5.56 & 0.00 & 5.56 \\
\hline$M K K 7^{E D}-5 A$ & 15.440 & 12.606 & 5.219 & 4.017 & 125 & 88.00 & 54 & 81.48 & 14.81 & 3.70 & 0.00 & 3.70 \\
\hline$M K K 7^{E D}-5 B$ & 7.238 & 4.545 & 1.317 & 0.200 & 198 & 16.67 & 60 & 0.00 & 48.33 & 40.00 & 11.67 & 51.67 \\
\hline N7-MKK5 ${ }^{\mathrm{DD}}$-C7 & 3.526 & 1.153 & 2.411 & 0.915 & 91 & 54.95 & 43 & 2.33 & 13.95 & 13.95 & 69.77 & 83.72 \\
\hline N5-MKK7 $7^{\mathrm{ED}}-\mathrm{C} 5$ & 4.724 & 2.876 & 1.439 & 0.673 & 51 & 72.55 & 46 & 0.00 & 71.74 & 28.26 & 0.00 & 28.26 \\
\hline MPK3, not shown & & & & & 49 & 0.00 & 50 & 0.00 & 82.00 & 18.00 & 0.00 & 18.00 \\
\hline MPK3 ${ }^{D 193 G / E 197 A}$ & & & & & 18 & 0.00 & 52 & 0.00 & 80.77 & 19.23 & 0.00 & 19.23 \\
\hline $\mathrm{MPK}^{\mathrm{T} 119 \mathrm{C}}$, not shown & & & & & 43 & 0.00 & 89 & 0.00 & 86.52 & 13.48 & 0.00 & 13.48 \\
\hline MPK6, not shown & & & & & 122 & 0.00 & 45 & 0.00 & 84.44 & 15.56 & 0.00 & 15.56 \\
\hline MPK6 ${ }^{\mathrm{D} 218 \mathrm{G} / \mathrm{E} 222 \mathrm{~A}}$ & & & & & 6 & 100.00 & 27 & 0.00 & 77.78 & 22.22 & 0.00 & 22.22 \\
\hline MPK6 ${ }^{\mathrm{Y} 144 \mathrm{C}}$, not shown & & & & & 35 & 0.00 & 21 & 0.00 & 90.48 & 9.52 & 0.00 & 9.52 \\
\hline
\end{tabular}

Kinase activity is determined in triplicates with the exception of those marked with "**" that were done in duplicates. Averages (AVG) and Standard Errors (SE) are represented in the table. Phenotypic categories for FAMA stage phenotypes are described in Methods

kinase fold [31] (Fig. 3a and b, with primary sequence in Additional file 3: Figure S3A). The core catalytic domains of MKK5 and MKK7 are quite similar, but the $\mathrm{N}$ - and $\mathrm{C}$-termini are variable. This is similar to MEK1/2, where catalytic domains are similar and well resolved, but the flanking $\mathrm{N}$ - and $\mathrm{C}$-terminal extensions and a region containing the PRS are not. In addition, MKK5 possesses, but MKK7 completely lacks, sequences at positions comparable to the C-terminal extension in MEK1/2 (Additional file 3: Figure S3C). Previously, we showed that this C-terminal extension does not contribute to MKK5 activity or specificity, but that N-termini have an important role in MKKs activities, possibly through the presence of $\mathrm{D}$-docking domains that mediate interactions with downstream MPKs [23].

In addition to the distinct $\mathrm{C}$-terminal distal region (CDR), divergent surface-exposed regions of the plant MKKs include a C-terminal proximal region (CPR) between conserved subdomains VIII and X (Fig. 3, Additional files 3 and 4: Figures S3 and S4). The CPR contains two loops. Loop A starts immediately downstream of the YM(S/A)PER sequence, a MKK signature [2], and ends before the highly conserved $\alpha$-helix F. The sequence of loop A is similar between kinases with identical functions and different between kinases with divergent functions in both Arabidopsis and humans (Additional file 4: Figure S4). For example, loop A in MKK4 and MKK5 is identical, whereas it differs between MKK5 and MKK7. Loop B is downstream of $\alpha$-helix F and displays a high tolerance for sequence variability (Fig. 3, Additional files 3 and 4: Figures S3 and S4). Among CMGC (Cyclin-dependent kinases, MAPK, Glycogen synthase kinase and Cyclin-dependent kinase-like kinase) group kinases, loop B contains an insert that binds interacting proteins [29], and a different insert in MEK1/2 mediates binding to MAPK scaffolds MP1 and KSR [15, 32]. This region is shorter in plant MKKs (making them resemble human MEK3-MEK7), but the sequence divergence among MKKs is consistent with this loop being a specificity or identity determinant. It is therefore a prime region to target in our dissection of specificity.

$\mathrm{N}$-termini link specific MKKs to specific phenotypes Different in vivo behaviors of MKK5 $5^{\mathrm{DD}}$ and MKK $7^{\mathrm{ED}}$ make it possible to begin to correlate unique sequences 


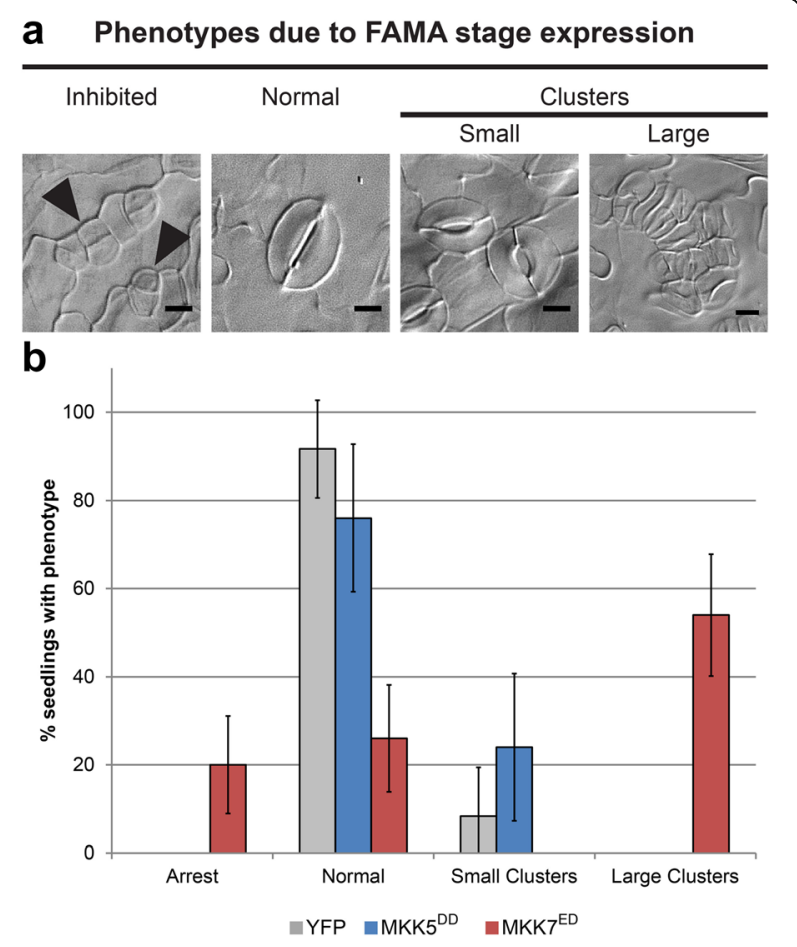

Fig. 2 Differences phenotypic output between MKK5 and MKK7. a Micrographs of phenotypes resulting from FAMA-stage expression of $M K K 5^{D D}$ and $M K K 7^{E D}$ : precursors exit the stomatal lineage and do not make GCs (arrowheads); Normal (single stomata comprised of two GCs) or small (2-3) or large (4+) clusters of adjacent stomata resulting from overproliferation of precursors before they become GCs. Scale bars are $10 \mu \mathrm{m}$. b Quantification of phenotypes, percentage of seedlings showing one of four phenotypes. >19 independent transformants were scored per genotype and stage (Ns reported in Table 1). 35Spro:YFP was used as a negative control (see Methods). Error bars correspond to $95 \%$ confidence intervals

and structures with unique functions. Informed by the structural analysis, we made chimeric MKKs based on dividing the MKKs into $\mathrm{N}$ - terminal $(\mathrm{N})$, kinase domain (MKK) and C-terminal (C) regions, and further dividing the $\mathrm{C}$ domain into $\mathrm{CDR}$ and $\mathrm{CPR}$ (and within CPR, Loop A and B) (Fig. 3). To assay the function of these domains, we measured the phenotypes induced by chimeras at the FAMA stage in T1 s and compared to those obtained for intact MKK5 ${ }^{\mathrm{DD}}$ and $\mathrm{MKK} 7^{\mathrm{ED}}$. Expression and subcellular localization of YFP-tagged MKKs was verified by confocal microscopy. We predicted that certain domain combinations could result in non-functional chimeras. Because expression of MKK5 ${ }^{\mathrm{DD}}$ (Fig. 4a) and non-functional chimeras would give essentially the same phenotype at the FAMA stage (no effect on stomatal development), it was important to discriminate $\mathrm{MKK} 5^{\mathrm{DD}}$ like chimeras from non-functional chimeras. We took two approaches to verify that kinases were still active. First, we measured the intrinsic kinase activity against MPK3/6 in in vitro kinase assays (Additional file 2: Figure S2).
Second, we took advantage of the fact that both $M K K 5^{\mathrm{DD}}$ and $M K K 7^{\mathrm{ED}}$ drive robust inhibition of stomatal initiation at the $\mathrm{SPCH}$ stage [22] to create an in vivo assay for kinase activity. We expressed the chimeras at the SPCH stage and quantified the degree of inhibition of stomatal initiation (Table 1).

To characterize the role of the N-terminus in MKK5, we replaced it with the N-terminus of MKK7 (N7-MKK $5^{\mathrm{DD}}$ ) and the chimera was expressed in FAMA stage cells. $59 \%$ of $\mathrm{T} 1$ transformants produced stomatal clusters (Fig. 4b and g), though clusters were smaller than those generated by $\mathrm{MKK} 7^{\mathrm{ED}}$. In addition, $9 \%$ of $\mathrm{T} 1$ transformants showed inhibition of stomatal formation (Fig. 4g). We also noticed that N7-MKK $5^{\mathrm{DD}}$ partially relocalized to mitochondria (Fig. $4 \mathrm{~b}$ ) similar to MKK $7^{\mathrm{ED}}$ [23]. Our in vitro and in vivo controls for activity both indicated that $\mathrm{N} 7-\mathrm{MKK} 5^{\mathrm{DD}}$ was less active than intact MKK $5^{\mathrm{DD}}$; only $\sim 27 \%$ of $\mathrm{T} 1 \mathrm{~s}$ inhibited stomatal initiation (Fig. 4h) and in vitro kinase activity was lower, especially towards MPK3 (Fig. 4i). This dramatic output alteration (aphenotypic MKK5 ${ }^{\mathrm{DD}}$ to a weak $\mathrm{MKK}^{\mathrm{ED}}$-like behavior) suggests that the $\mathrm{N}$-terminus is more than just a structural/ regulatory region required for protein activity. Instead, it appears to channel the MKK towards specific phenotypic outcomes. This specificity behavior resembles that observed in yeast where MKKs involved in other cellular processes were engineered to interact with components of the mating pathway, but were only able to transduce a mating signal when their $\mathrm{N}$-termini were replaced with the $\mathrm{N}$-terminus from Ste7, the mating specific MKK [24].

If the N-terminus enforces MKK specific activities, then replacement of N7 by N5 in MKK ${ }^{\mathrm{ED}}$ should reveal the endogenous response to MKK5 activation. With the N5-MKK $7^{\mathrm{ED}}$ chimera we found efficient inhibition of lineage initiation at the $\mathrm{SPCH}$ stage and a normal ability to phosphorylate MPK3 and MPK6 in vitro (Fig. 4h and i). Like MKK5 ${ }^{\mathrm{DD}}$ the chimera was cytoplasmic localized (compare $\mathrm{A}$ and $\mathrm{C}$ in Fig. 4). Unlike $\mathrm{MKK} 5^{\mathrm{DD}}$, N5MKK7 ${ }^{\mathrm{ED}}$ completely inhibited GC production (Fig. 4c and g). Thus, with this manipulation, we were finally able to recapitulate the stomatal lineage inhibition phenotype we had expected from $M K K 5^{\mathrm{DD}}$ based on its ability to inhibit stomatal development at earlier stages [22] and the loss of function stomatal cluster phenotype [21].

\section{Loop B prevents MKK5 ${ }^{\mathrm{DD}}$ from inhibiting stomata formation at the FAMA stage}

Demonstrating that development of FAMA-stage cells could be inhibited, however, raised the question of why intact $M K K 5^{\mathrm{DD}}$ is unable to do so. We hypothesized that sequences in the MKK5 C-terminus act as negative regulatory regions. To test this idea, we first replaced the entire C-terminal region of MKK5, creating $\mathrm{MKK}^{\mathrm{DD}}{ }_{-} \mathrm{C} 7$. FAMA-stage expression did result in a partially penetrant 

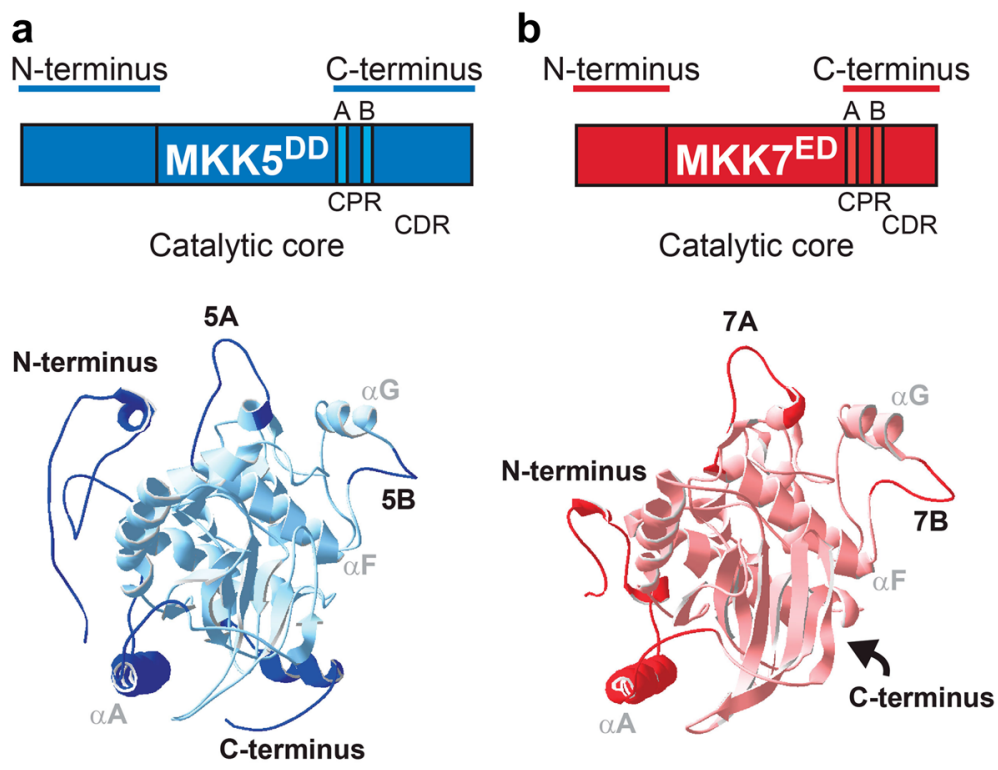

Fig. 3 Differences in protein structure between MKK5 and MKK7. a-b Schematic and predicted structures of MKK5 (blue) and MKK7 (red). CPR, C-terminal proximal region. CDR, C-terminal Distal Region. Four regions important for this study (N-termini, Loop A, Loop B and the C-termini) are bolded. Conserved a helices $A, F$ and $G$ are labeled in grey

inhibition of stomatal formation where inhibited precursors coexisted with normal stomata (Fig. $4 \mathrm{~d}$ and g). MKK $5^{\text {DD }}$-C7 displayed high activity in SPCH-stage lineage inhibition (Fig. 4h), but was less efficient than $\mathrm{MKK}^{\mathrm{DD}}$ in in vitro kinase assays, particularly towards MPK3 (Fig. 4i). Because previously reported MKK5 deletions in the CDR portion of the C-terminus did not significantly change MKK $5^{\mathrm{DD}}$ activities [23], we reasoned that putative regulatory regions were located in the CPR.

The largest sequence differences between $\mathrm{C} 5$ and C7 reside in loop A and B in the CPR. Substitution of loop A $\left(\mathrm{MKK}^{\mathrm{DD}}-7 \mathrm{~A}\right)$ resulted in a chimera that did not affect stomatal development at the FAMA stage (Fig. 4e and g), but substitution of loop $\mathrm{B}\left(\mathrm{MKK}^{\mathrm{DD}}-7 \mathrm{~B}\right)$ led to inhibition of stomatal formation at high frequency (Fig. $4 \mathrm{f}$ and g). This result suggests that MKK5's loop B is a region that blocks MKK5 from participating in stomatal inhibition at the FAMA stage. Interestingly, $\mathrm{SPCH}$ stage activity was markedly reduced for both chimeras (Fig. 4h), but in vitro activities of MKK $5^{\mathrm{DD}}$ $7 \mathrm{~A}$ and $\mathrm{MKK} 5^{\mathrm{DD}}-7 \mathrm{~B}$ were at least as high as that of MKK $5^{\mathrm{DD}}$ (Fig. 4i). These observations suggest that MKK $5^{\mathrm{DD}}-7 \mathrm{~A}$ and $\mathrm{MKK} 5^{\mathrm{DD}}-7 \mathrm{~B}$ are catalytically active kinases but cannot generate appropriate signals in vivo.

\section{Loop $B$ is required for robust $M K K 7^{\mathrm{ED}}$ activity}

If there was truly a discrete domain of MKK5 that was subject to negative regulation, then transferring it to $M K K 7^{\mathrm{ED}}$ should dampen the stomatal clustering phenotype at the FAMA stage (Fig. 5a). We initially swapped the entire $\mathrm{C}$-terminus, and the resulting $\mathrm{MKK} 7^{\mathrm{ED}}-\mathrm{C} 5$ only produced normal stomata, similarly to MKK5 ${ }^{\mathrm{DD}}$ (Fig. $5 \mathrm{~b}$ and g). This could suggest that C5 is able to block MKK7 inhibitory function at FAMA stage. However, monitoring other indicators of MKK activity (SPCH stage lineage inhibition and in vitro phosphorylation of MPK3 and MPK6) suggested that $\mathrm{C} 7$ was essential for overall activity (Fig. $5 \mathrm{~h}$ and i). Thus this phenotype is the result of creating a generally inactive MKK7 chimera, more than an effect due to the presence of MKK 5 regulatory sequences. Thus, we split C5 into CDR5 and CPR5, and determined if we could restore $\mathrm{MKK}^{\mathrm{ED}}$ activity in vitro and in the $\mathrm{SPCH}$ stage. Activity was restored in $\mathrm{MKK} 7^{\mathrm{ED}}$-CDR5: this chimera completely inhibited lineage initiation at the SPCH stage and was indistinguishable from $\mathrm{MKK} 7^{\mathrm{ED}}$ in in vitro kinase assays (Fig. $5 \mathrm{~h}$ and i). Rather than decreasing $M K K 7^{\mathrm{ED}}$ function, however, $M K K 7^{\mathrm{ED}}$-CDR5 had a strikingly stronger inhibitory effect on stomatal development at the FAMA stage than MKK $7^{\mathrm{ED}}$ (Fig. $5 \mathrm{c}$ and g). In fact, it resembled the strong phenotype produced by N5-MKK $7^{\mathrm{ED}}$ (Fig. 4c). This implies that CDR5, like N5, channels MKK activity to inhibition of stomatal development. In contrast, MKK $7^{\mathrm{ED}}$-CPR5 was largely inactive at both SPCH and FAMA stages (Fig. 5d and g), and in in vitro phosphorylation assays against MPK3 and MPK6 (Fig. 5h and i), indicating that CPR7 is necessary for MKK $7^{\mathrm{ED}}$ catalytic activity.

So far, the domains from MKK5 that dampened MKK $7^{\mathrm{ED}}$ activity at the FAMA stage also decreased the activity of the chimeras in vitro and at the $\mathrm{SPCH}$ stage $\left(\mathrm{MKK}^{\mathrm{ED}}\right.$-C5 and MKK7 ${ }^{\mathrm{ED}}$-CPR5). From these results, it appears that the CPR region is important for MKK $7^{\mathrm{ED}}$ 

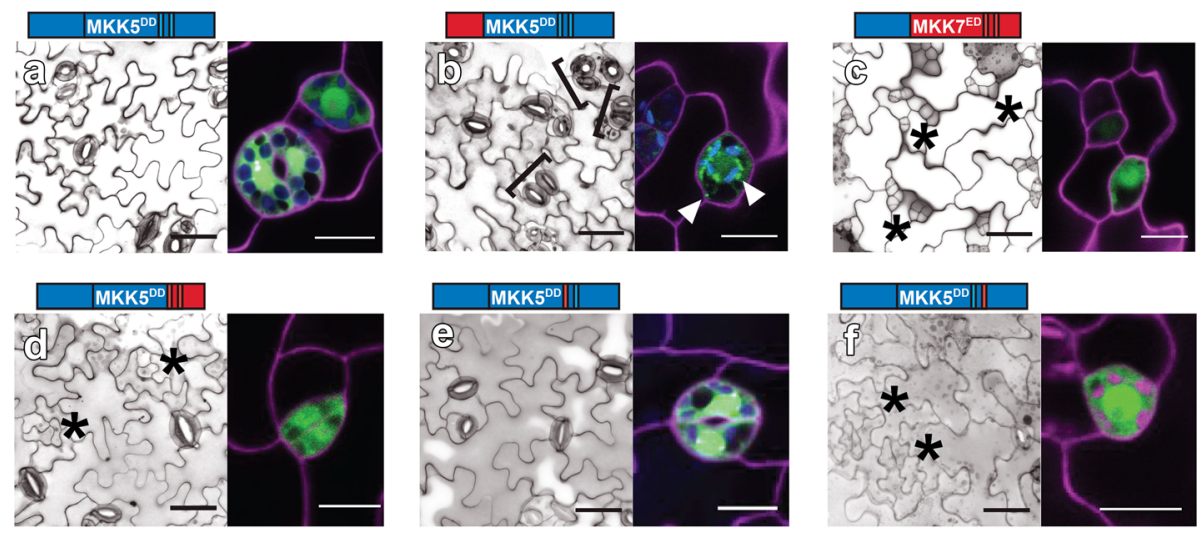

g

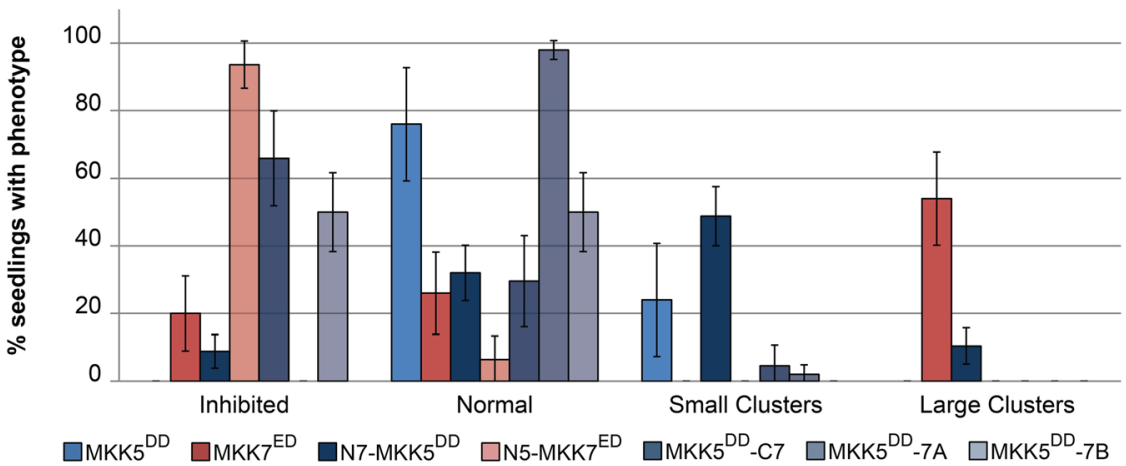

h

i
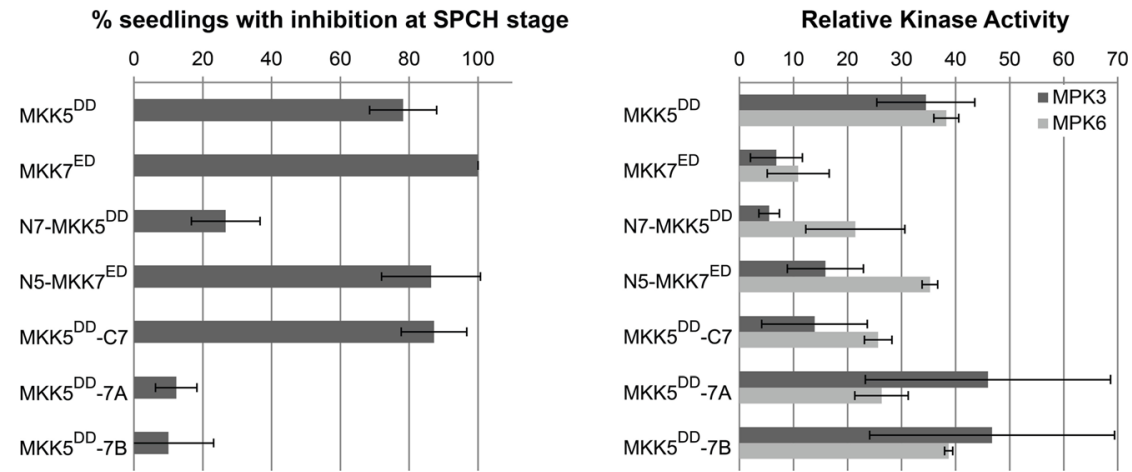

Fig. $4 \mathrm{~N}$-termini link MKKs to their phenotypic outputs and the C-terminal Loop B is required for FAMA stage-specific regulation of MKK5. a-f Paired micrographs of representative major phenotype (left) and subcellular localization (right), of specific MKK5 ${ }^{\mathrm{DD}}$ - and MKK7 ${ }^{\mathrm{ED}}$-YFP variants (diagramed above). Black brackets mark clusters and asterisks indicate inhibition. YFP is in green and cell outlines in magenta. For example, in (a) MKK5 ${ }^{\mathrm{DD}}$ expression results in a WT phenotype and the protein is cytoplasmic and in (b) mitochondrial/cytoplasmic N7-MKK5 ${ }^{\mathrm{DD}}$ induces stomatal clustering. Mitochondria-localized signals indicated with white arrowheads. Scale bars are $50 \mu \mathrm{m}$ in phenotype images and $10 \mu \mathrm{m}$ for localization images. $\mathbf{g}$ Quantification of phenotypes in (a-f). $\mathbf{h}$ SPCH stage inhibition of lineage initiation. Error bars in (g) and (h) correspond to $95 \%$ confidence interval. $\mathbf{i}$ In vitro kinase activity towards kinase inactive MPK3 and MPK6. Kinase assays were performed in triplicates, normalized to unphosphorylated KI-MPK and averaged; error bars represent standard errors

catalytic activity and thus we created smaller domain swaps (loops $\mathrm{A}$ and $\mathrm{B}$ ) to attempt to transfer negative regulatory sequences from MKK5 into $\mathrm{MKK} 7^{\mathrm{ED}}$ without affecting kinase functionality. $\mathrm{MKK} 7^{\mathrm{ED}}-5 \mathrm{~A}$ and $\mathrm{MKK}^{\mathrm{ED}}-5 \mathrm{~B}$ were active in vivo (inhibited lineage initiation at the $\mathrm{SPCH}$ stage, Fig. 5h) and in vitro (phosphorylated MPK3/6, Fig. 5i), although to different degrees. At the FAMA stage, $M K K 7^{\mathrm{ED}}-5 \mathrm{~A}$ inhibited stomatal formation to a greater extent than $M K K 7^{\mathrm{ED}}$ (Fig. 5e and g). This behavior is similar to $\mathrm{N} 5-\mathrm{MKK}^{\mathrm{ED}}$ (Fig. 4c) and $\mathrm{MKK}^{\mathrm{ED}}{ }_{-\mathrm{CDR}}$ (Fig. 5c), suggesting that N5, loop 5A and CDR5 restrict MKK $7^{\mathrm{ED}}$ activity to inhibition of stomatal development. In contrast, $\mathrm{MKK} 7^{\mathrm{ED}}-5 \mathrm{~B}$ 's ability to cause stomatal clustering and inhibition of stomatal development at 

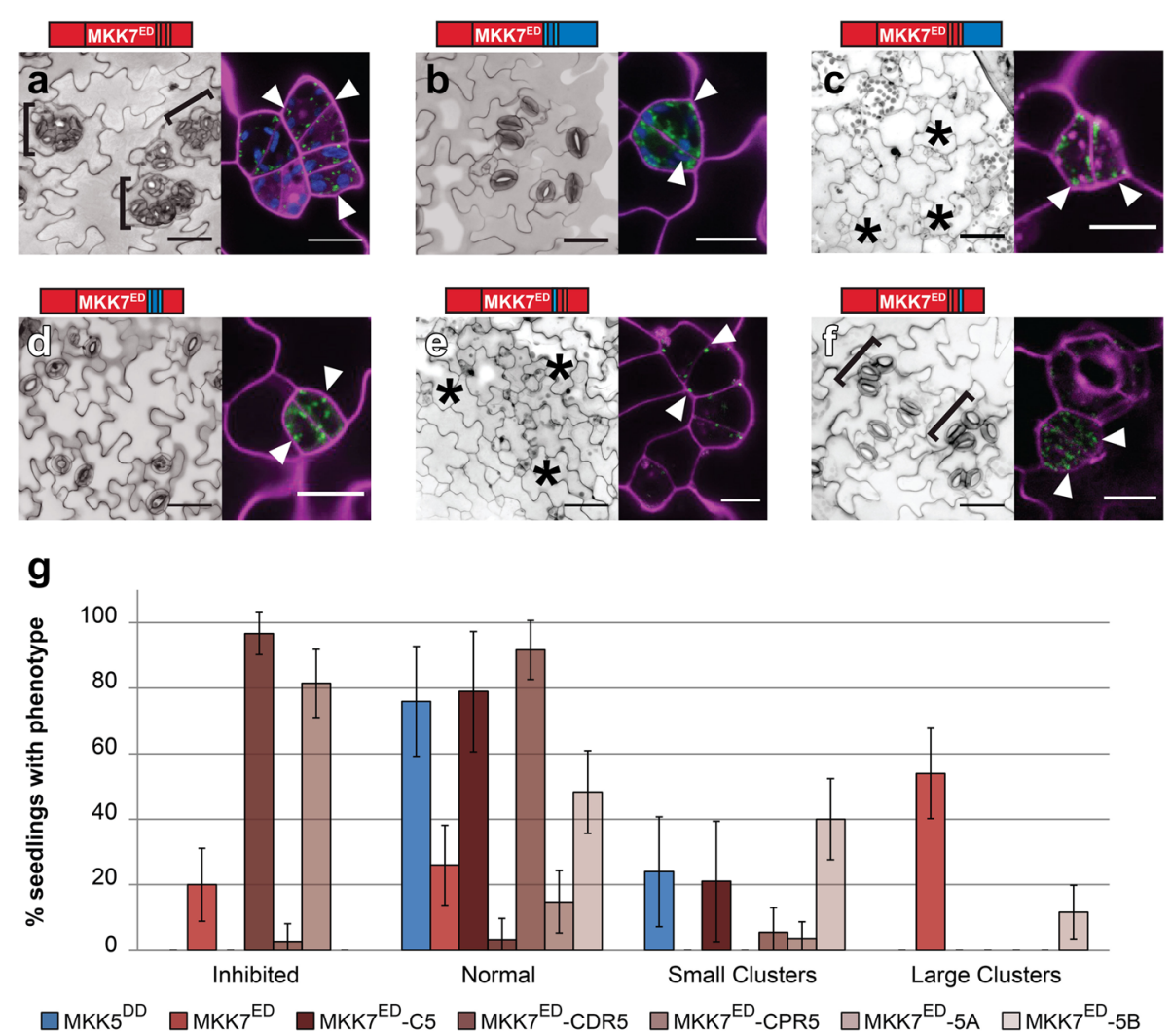

h

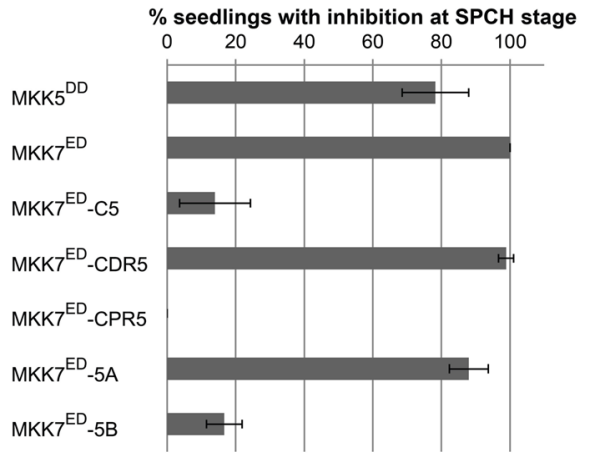

i

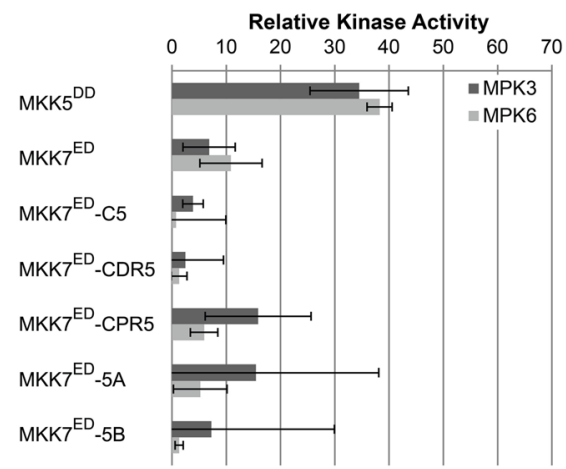

Fig. 5 Loops A and B are required for specific and robust FAMA stage MKK7 activities. a-f Paired micrographs of representative major phenotype (left) and subcellular localization (right), of specific MKK $7^{\mathrm{ED}}$-YFP variants diagramed above. Black brackets mark clusters and asterisks indicate inhibition. YFP is in green and cell outlines in magenta; white arrowheads point to mitochondrial localization. For example, in (a) MKK $7^{\mathrm{ED}}$ induces stomatal clustering (brackets) and is mitochondrial localized (white arrowheads). Scale bars are $50 \mu \mathrm{m}$ for phenotype images and $10 \mu \mathrm{m}$ for localization images. $\mathbf{g}$ Quantification of phenotypes in $(\mathbf{a}-\mathbf{f})$. $\mathbf{h}$ SPCH stage inhibition of lineage initiation. Error bars in $(\mathbf{g})$ and (h) correspond to $95 \%$ confidence interval. i In vitro kinase activity towards kinase inactive MPK3 and MPK6. Kinase assays were performed in triplicates, normalized to unphosphorylated KI-MPK and averaged; error bars represent standard errors

the FAMA stage was markedly reduced when compared to $M K K 7^{\mathrm{ED}}$ (Fig. 5g). Because MKK $7^{\mathrm{ED}}-5 \mathrm{~B}$ also showed reduced activities in other indicators of MKK activity (Fig. $5 \mathrm{~h}$ and i), we concluded that the negative regulation of $\mathrm{MKK} 5^{\mathrm{DD}}$ is restricted to loop $5 \mathrm{~B}$ but cannot be transferred without affecting $M K K 7^{\mathrm{ED}}$ catalytic activity. Nevertheless, the same results highlight that loop $7 \mathrm{~B}$ is required for robust $\mathrm{MKK} 7^{\mathrm{ED}}$ activity.

\section{Swapping domains allows specificity to be changed in} MKK5 $^{\mathrm{DD}}$ and MKK $7^{\mathrm{ED}}$

Our results show that the $\mathrm{N}$-terminus, CPR region (loops $A$ and $B$ ) and $C D R$ region modulate MKK activity. We 
showed that N7 and CPR7 are necessary for $M K K 7^{\mathrm{ED}}$ mediated GC clustering at the FAMA stage, but when CDR5 is incorporated into MKK $7^{\mathrm{ED}}$, GC production is inhibited. If our "wiring diagram" for specificity is correct, then a chimera that contains the GC promoting domains from MKK7 but not the inhibitory CDR5 (i.e., N7$M K K 5^{\mathrm{DD}}$-C7) should mimic MKK $7^{\mathrm{ED}}$. Indeed, when we constructed N7-MKK $5^{\mathrm{DD}}-\mathrm{C} 7$, it resembled MKK ${ }^{\mathrm{ED}}$ both qualitatively and quantitatively (Fig. 6a and c). Likewise, N5-MKK $7^{\mathrm{ED}}-\mathrm{C} 5$ should match MKK ${ }^{\mathrm{DD}}$ activities, and it does in planta (Fig. $6 \mathrm{~b}$ and c). Interestingly, robust rewiring in vivo (Fig. 6c and d) appears to be uncoupled from kinase activity in vitro, as both rewired proteins were much less capable of phosphorylating MPK3 and MPK6 than MKK5 ${ }^{\mathrm{DD}}$ and MKK $7^{\mathrm{ED}}$ (Fig. 6e). One interpretation of these swaps is that specificity lies only outside of the kinase domain. If this were true, then we should be able to generate a chimera that resembles $\mathrm{N} 7-\mathrm{MKK} 5^{\mathrm{DD}}-\mathrm{C} 7$ and $M K K 7^{\mathrm{ED}}$ using the kinase domain from another MKK. We selected the kinase domain of MKK6 that can also phosphorylate MPK3 and MPK6 in vitro [25], and created N7-MKK $6^{\mathrm{DD}}-\mathrm{C} 7$. Expression of N7-MKK6 ${ }^{\mathrm{DD}}-\mathrm{C} 7$ at the FAMA stage, however, did not produce any noticeable phenotype (Additional file 5: Figure S6). This suggests that although kinase domains in MKK5 and MKK7 are not differential, they still contain stomatal fate-enabling regions.
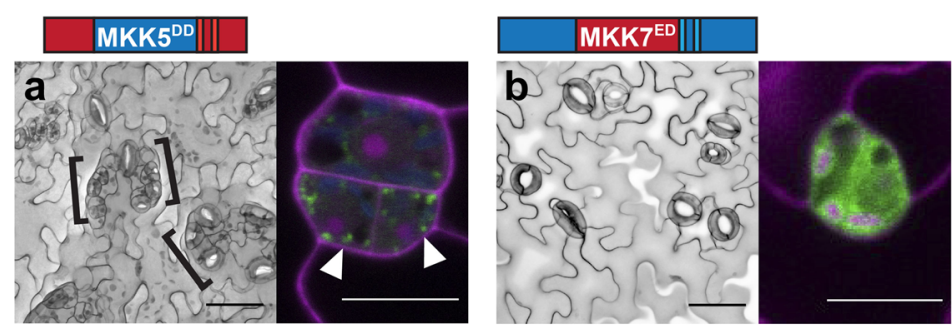

C
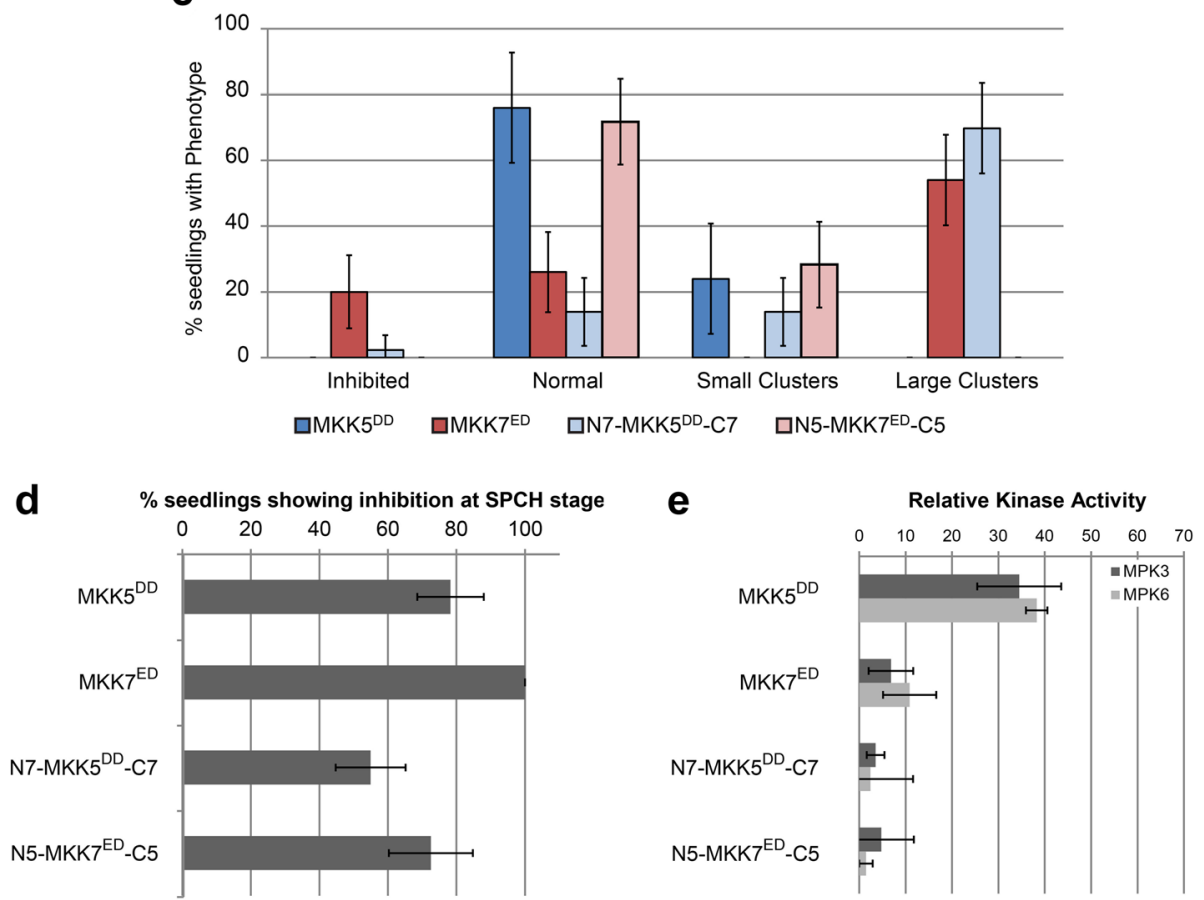

Fig. 6 MKK5 and MKK7 activity and localization in the stomatal lineage can be reciprocally rewired. a-b Paired micrographs of representative major phenotype (left) and subcellular localization (right), specific MKK-YFP variants diagramed above. Black brackets mark clusters; YFP is in green and cell outlines in magenta; white arrowheads point to mitochondrial localization. a N7-MKK5 ${ }^{\mathrm{DD}}-\mathrm{C} 7$ mimics $\mathrm{MKK} 7^{\mathrm{ED}}$ in that it produces clusters and can localize to mitochondria. b N5-MKK $7^{\mathrm{ED}}-\mathrm{C} 5$ mimics MKK5 ${ }^{\mathrm{DD}}$ in that it has a WT phenotype and localizes in the cytoplasm. Scale bars are $50 \mu \mathrm{m}$ in phenotype images and $10 \mu \mathrm{m}$ for localization images. c Quantification of phenotypes (a-b). d SPCH stage inhibition of lineage initiation. Error bars in (c) and (d) correspond to 95\% confidence interval. e In vitro kinase activity towards kinase inactive MPK3 and MPK6. Kinase assays were performed in triplicates, normalized to unphosphorylated KI-MPK and averaged; error bars represent standard errors 


\section{Comprehensive analysis of chimeras reveals functions of MKK domains}

We repeatedly observed that the ability of native and chimeric MKKs to phosphorylate their targets in vitro does not predict their activities in vivo. In fact, when chimera data are considered together, in vitro versus in vivo data have no statistical correlation (Additional file 6: Figure S7). In contrast, when only in vivo data were compared, activities in SPCH and FAMA stages were positively correlated (Fig. 7a). Interestingly, native and chimeric MKKs were distributed in two subpopulations. MKKs closer to the regression line promoted stomatal clustering (red dots) or inhibited stomatal formation (black dots). MKKs further from the regression line had no effect in stomatal development at the FAMA stage (blue dots), but had a broad range of activities at the $\mathrm{SPCH}$ stage (shaded area in Fig. 7a). This behavior might be reflecting the additional regulation that some of the MKKs showed at the FAMA stage.

We reasoned that MKKs subject to the same regulation would share structural similarities. To test this hypothesis and generate an overall picture of the relationship between MKK structural domains and in vivo functions, we clustered 15 native and chimeric MKKs and controls according to their quantitative phenotype data at the FAMA stage (clustering detailed in methods). Constructs robustly fell into three clusters (Fig. 7b): Cluster 1, no phenotypic effect; Cluster 2, induces stomatal proliferation; and Cluster 3, inhibits stomatal formation (the presumed endogenous role for MKK5). Within clusters, however, not all MKKs were identical. We performed sequential tests of independence to determine how similar the distribution of phenotype frequencies was between chimeras in each cluster. Cluster 1 was composed of MKKs similar to MKK5 ${ }^{\mathrm{DD}}$ (group 1) and MKKs similar to inactive YFP (group 2). Cluster 2 was statistically separated into weak $M K K 7^{\mathrm{ED}}$-like chimeras (group 3) and two MKKs that induced strong clustering, yet were different from each other. Cluster 3 was statistically separated into strong inhibitors of stomatal formation (group 4) and weak inhibitors (different from each other).

To summarize, when analyzing native and chimeric MKK structures across Clusters, we see that loops A and $B$ have discrete functions in selecting MKK-specific outputs and in kinase activity in vivo. Loop A can be thought of as a "channel selector", that, together with $\mathrm{N}$-terminus and CDR, selects between the normal role of arresting stomatal progression and the artificial role of promoting stomatal clustering. Loop B is a "volume control" with the $7 \mathrm{~B}$ version increasing, and $5 \mathrm{~B}$ decreasing, the phenotypes specified by the other domains of the MKKs.

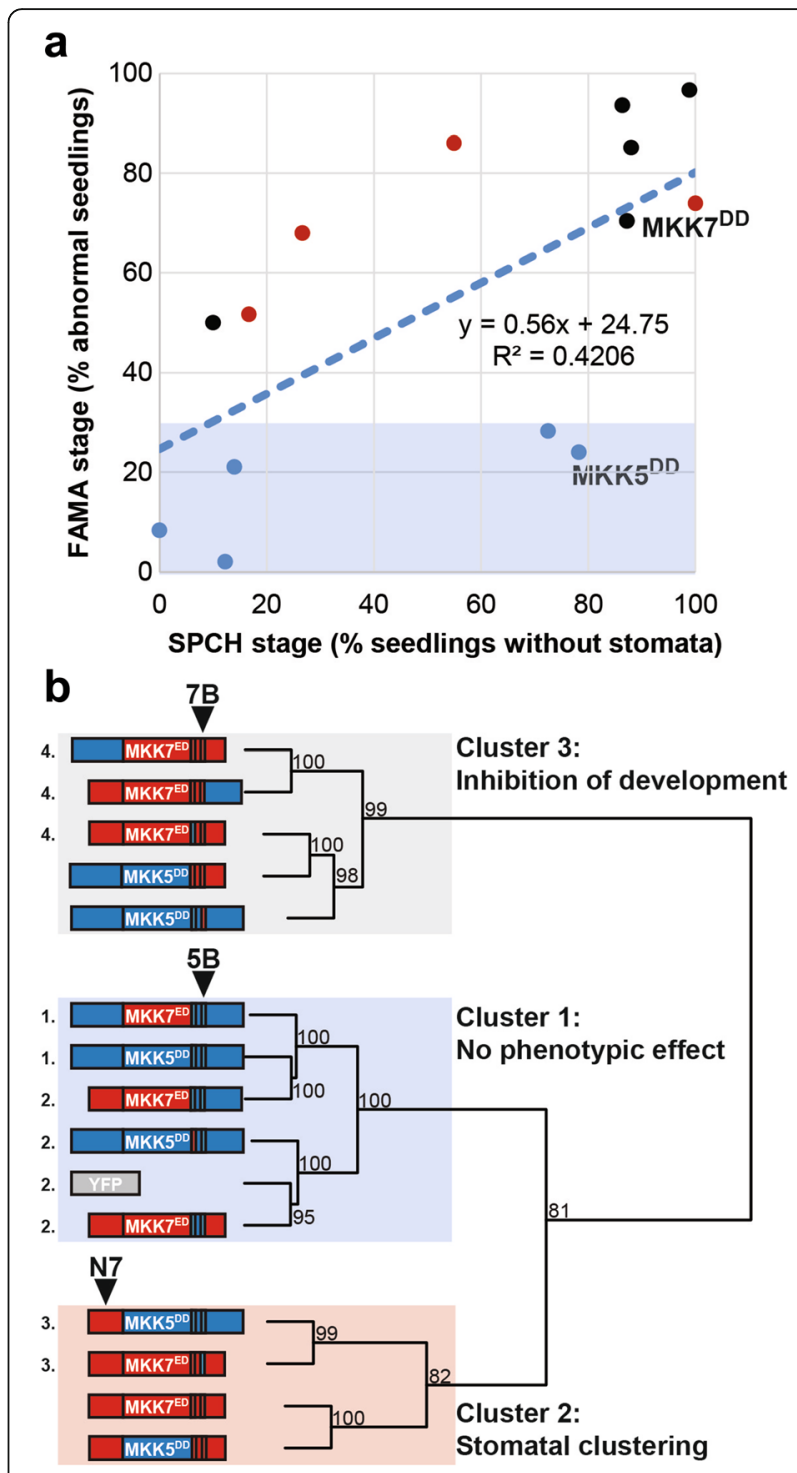

Fig. 7 MKK5 defines a cluster of chimeras with low activity at FAMA stage, whereas clustering and inhibiting chimeras group in two independent clusters. a SPCH and FAMA stage activities show a weak positive correlation. Shaded area corresponds to activities at the FAMA stage lower than $30 \%$. Data points are colored according to the cluster they belong to in (b). MKK activities at the FAMA stage are separated into three clusters (b): cluster 1 contains MKKs that show no phenotypic effect and contain loop 5B; cluster 2 contains MKKs that lead to stomatal clustering and contain N7; and, cluster 3 contains MKKs that lead to inhibition of stomatal development and contain loop 7B. Numbers next to nodes correspond to approximately unbiased $p$-values with bootstrap replications set to 1000 . Chimeras not significantly different in Chi-squared tests if independence are indicated with an identical number $(p>0.05)$

MPK6 mediates GC inhibition, but like MKK5, is prevented from doing so at the FAMA stage

Previous loss- and gain-of-function experiments placed MPK3 and MPK6 downstream of an activated MKK4/5 homologue (NtMEK2), suppressing stomatal formation 
[21] in the early stages of the stomatal lineage, but this assay could not address the potential for MPK3 and MPK6 to mediate FAMA-stage activities. Our chimeras that drive stomatal inhibition at the FAMA-stage, however, could be used to see whether either mediated such late stage inhibition. We used N5-MKK ${ }^{\mathrm{ED}}$ which, in WT led to complete inhibition of stomatal development (Fig. 8a, Table 1). When expressed in the loss of function mpk6-3 background, N5-MKK7 ${ }^{\mathrm{ED}}$ failed to promote complete inhibition in 19 independent T1 s (Fig. 8b) indicating that MPK6 is likely downstream. This led us to the question of whether MPK6, like MKK $5^{\mathrm{DD}}$, would also be actively inhibited from effecting fate at the FAMA stage. To test this, we created a constitutively active MPK6 $\left(\mathrm{MPK6}^{\mathrm{DE}}\right)$ [33] and tested its ability to suppress stomatal formation. Expression of $\mathrm{MPK} 6^{\mathrm{DE}}$ (but not $\mathrm{MPK}^{\mathrm{DE}}$; Fig. 8c, Table 1) inhibited stomatal progression at the $\mathrm{SPCH}$ stage, indicating that $\mathrm{MPK} 6^{\mathrm{DE}}$ is active in this assay. When expressed at the FAMA stage, however, $M P K 6^{\mathrm{DE}}$ did not affect stomatal development
(Fig. 8d, Table 1), a phenotypic output remarkably similar to that of $M K K 5^{\mathrm{DD}}$ (early, but not late, inhibition). We hypothesized that MKK5 and MPK6 normally repress stomatal development, but are actively prevented from having this effect at the FAMA stage.

\section{Discussion}

In multicellular organisms, coordinated development requires constant communication between cells and the evaluation of environmental conditions. All this information is integrated to decide from a spectrum of possible outputs, and the spectrum is frequently limited by a cell's identity. In previous, more superficial studies, FAMA stage cells appeared to lose the ability to inhibit stomatal development upon MAPK activation [22]. Here we show that these cells do not lack the capacity to be inhibited, but rather that MKK5 (and possibly MPK6) may be actively prevented from participating in this cellular outcome. Interestingly, from a cellular behavior perspective, the inhibition of stomatal formation appears
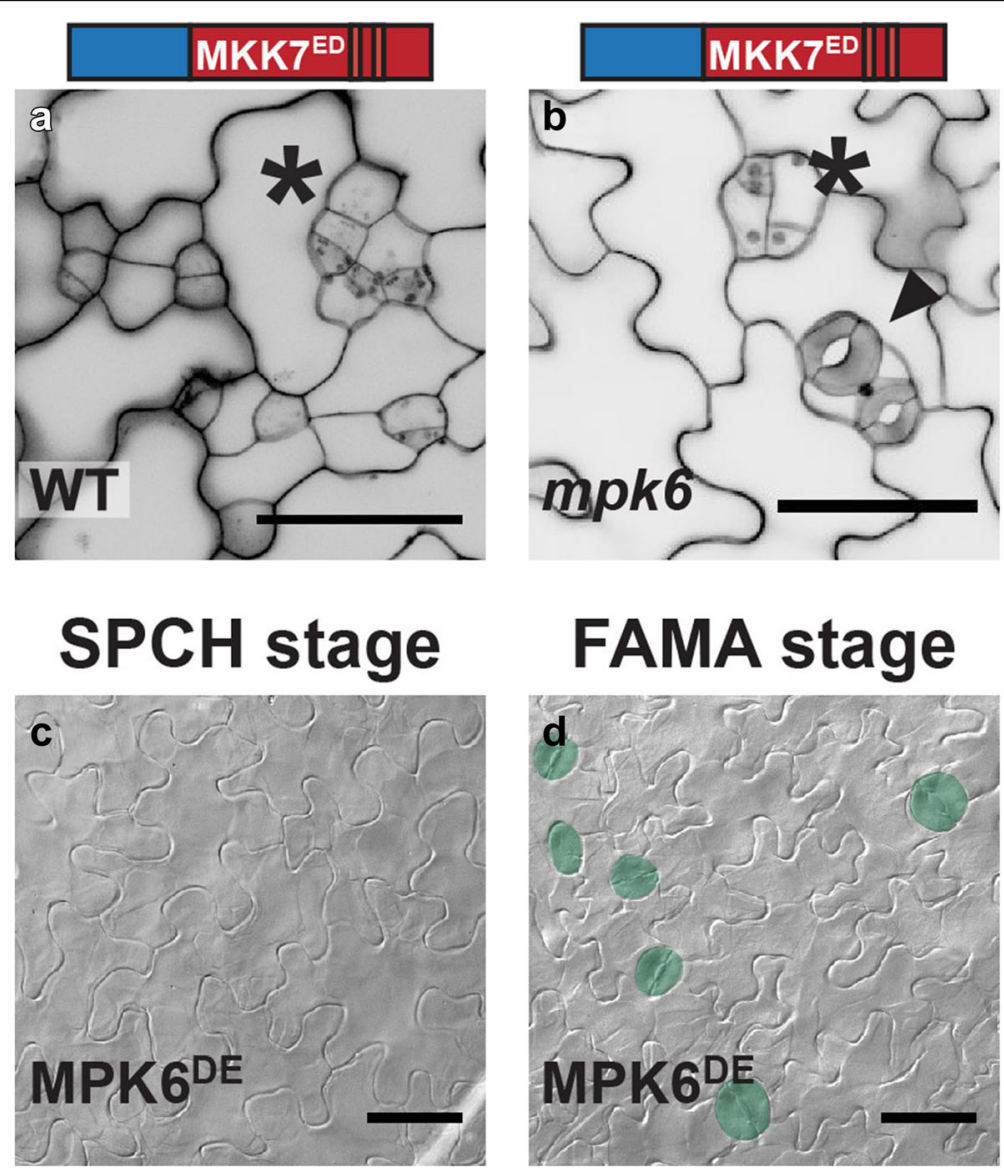

Fig. 8 MPK6 inhibits stomatal development at the FAMA stage in a MKK-specific manner. a and b Micrographs indicating the requirement for MPK6 as a downstream factor in N5-MKK ${ }^{\text {ED }}$-mediated FAMA-stage inhibition (asterisks). In mpk6 mutants (b), stomata (arrowhead) coexist with precursors that exit the lineage. (c and $\mathbf{d}$ ) DIC micrographs showing results of expression of MPK6 ${ }^{\mathrm{DE}}$ at SPCH and FAMA stages, stomata are highlighted in green. MPK3 ${ }^{\text {DE }}$ does not affect development at any stage (Table 1). Scale bars in are $50 \mu m$ 
to be due to developmental reprogramming and not an ROS-induced cell death (a response induced by CAMKKs in other contexts [34, 35]). In none of our analyses of leaves at seedling or mature plant stages did we detect lesions typical of cell death, nor did we observe the increased autofluorescence indicative of a burst of ROS production. Seedling lethality resulting from MKK manipulations under SPCH or FAMA promoters is likely a result of carbon dioxide starvation due to lack of functional stomata as these seedlings exhibit the pale, thin leaves common in stomataless mutants [36].

Structural analysis and engineered chimeras revealed that this regulation and other specific responses rely on distinct MKK domains. We found that Arabidopsis MKKs behave as modular proteins with four discrete regions: N-terminus, CDR and two loops (A and $\mathrm{B}$ ) in the CPR. N-termini contribute to subcellular localization (Fig. 4 and in [23]), to phenotypic output (Fig. 4) and may mediate interactions with downstream MPKs through their docking domains. In particular, we hypothesize that N7 has the ability to bind different types of MPK. Throughout development, MKK7 inhibits stomatal development by recruiting MPK3/6, but a yet unknown proliferative MPK mediates stomatal clustering at the FAMA stage (Fig. 9a). In the C-termini, Arabidopsis MKKs 1, 2, 4, 5 and 6 contain an extension that could be equivalent to the MKKK-interacting domain for versatile docking (DVD) in human MEKs 1, 3, 4, 6 and 7 (Additional file 4: Figure S4) [37]. Arabidopsis MKK7, 8,9 , and 10 , however, lack this domain, making it unclear how they engage the appropriate MKKK. In fact, the addition of CDR5 to MKK $7^{\mathrm{ED}}$ restricted this kinase's activity to an inhibitory output (Fig. $5 \mathrm{c}$ and g), suggesting that CDR5 interferes with $\mathrm{MKK} 7^{\mathrm{ED}}$ interactions. Upstream of the CDR, Loop A and B are two surface-exposed modules in the CPR that may contribute to establishing interactions with other network components. In our experiments, swapping loop $\mathrm{A}$ in $\mathrm{MKK} 7^{\mathrm{ED}}$ restricted its phenotypic output such that $\mathrm{MKK}^{\mathrm{ED}}-5 \mathrm{~A}$ only inhibited stomata formation (Fig. 5e, g). We propose then, that loop A promotes certain MKK-MPK interactions or, alternatively, restricts how MPKs contact MKKs. This hypothesis is supported by sequence similarities between human MEKs that share the same downstream MAPKs [1]. For example, ERK kinases MEK1 and MEK2 have identical loop As, and p38 kinases MEK3 and MEK6 differ at only one site (Additional file 4: Figure S4). Interestingly, human MEK7, which can phosphorylate both JNK and p38, shares some residues with MEK3 and MEK6 and others with the JNK kinase MEK4.

The function of loop B seems to be associated to MKKspecific regulation. Our data shows that loop B is required for robust $\mathrm{MKK}^{\mathrm{ED}}$ activity, but it prevents $\mathrm{MKK} 5^{\mathrm{DD}}$ mediated inhibition at the FAMA stage (Figs. 4f-g and

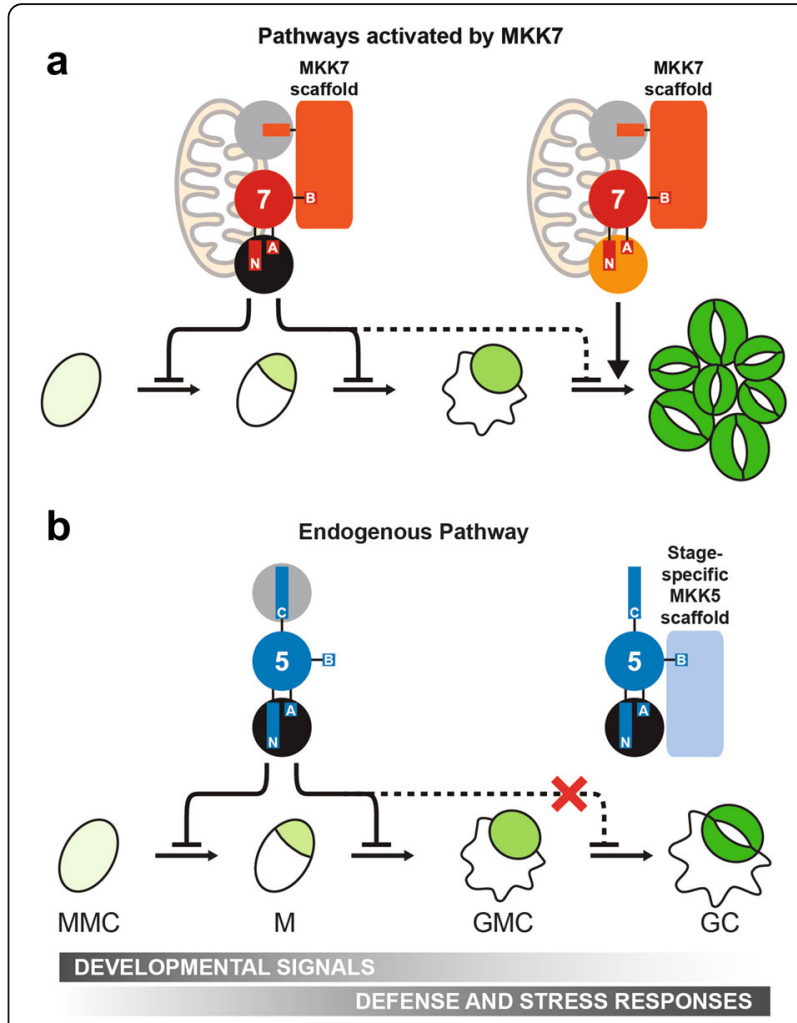

Fig. 9 A new model derived from activities during stomatal development for the endogenous MKK5 pathway and another activated by MKK7. a In the MKK7 pathway, MKK7 mimics MKK5 in early development, forcing precursors to exit the stomatal lineage. At the last stage, MKK7 induces two phenotypes. Firstly, MKK7 induces stomatal clustering by means of an unknown MKK7-specific MPK (orange circle). This proliferation depends on MKK7 mitochondrial Iocalization [23]. Secondly, MKK7 forces precursors to exit the lineage by escaping MKK5-specific regulation. An MKK7 scaffold enforces interactions between MKK7 and other components (MPKs and YODA, gray circles) within the network. $\mathbf{b}$ In the endogenous pathway, MKK5 and MPK3/6 (black circles) are involved in transducing developmental signals that inhibit stomatal lineage initiation and GMC commitment, and initiating defense and stress responses. At the last stage of development, a stage-specific MKK5 scaffold prevents MKK5 (and possibly MPK3/6) from inhibiting stomatal formation, only allowing defense and stress responses

$5 \mathrm{f}-\mathrm{g})$. Based on these phenotypes, we propose that loop B mediates interactions with different scaffolds (Fig. 9). A signal-promoting scaffold binds loop $7 \mathrm{~B}$ and enforces MKK7 interactions with its cognate MKKK and MPK (Fig. 9a). Such a scaffold would also explain why in vivo activity of $\mathrm{MKK} 7^{\mathrm{ED}}$ was always stronger than that of MKK $5^{\mathrm{DD}}$, even though in vitro assays showed an opposite pattern. On the other hand, we predict that a distinct scaffold recruited by loop $5 \mathrm{~B}$ prevents $\mathrm{MKK} 5^{\mathrm{DD}}$ from inhibiting stomatal development at the FAMA stage (inhibitory scaffold) (Fig. 9b). This prediction could also partially explain the behavior of certain chimeras. For example, the inhibitory scaffold would bind $M K K 7^{\mathrm{ED}}-5 \mathrm{~B}$ 
through loop 5B and dampen MKK $7^{\mathrm{ED}}$ activities. Likewise, a signal-promoting scaffold would bind $\mathrm{MKK} 5^{\mathrm{DD}}-7 \mathrm{~B}$ through loop 7B, releasing the inhibition of $M K K 5^{\mathrm{DD}}$.

The existence of signal-enhancing and inhibiting MAPK scaffolds in plants is supported by recent findings [38-40]. While the relevance of the first type is quite intuitive, the second type is more controversial. The inhibitory scaffold EDR1 was identified in the context of pathogen defense. Current hypotheses are that EDR1 provides a failsafe against inadvertently activating defense or cell death programs when other (not pathogeninduced) cues activate MAPK signaling. EDR modulates MPK3 activity indirectly by interacting with MKK $4 / 5$ and regulating their abundance [38]. Interestingly, we observed in FAMA stage cells that MPK6 was only inhibited when the upstream MKK was also inhibited. The clearest evidence that MKK5-MPK3/6 are scaffolded in FAMA stage cells would be, of course, to identify the scaffold. We tested whether EDR1 worked to inhibit FAMA-stage expressed $M K K 5^{\mathrm{DD}}$, however we failed to see a loss of inhibition in edr1 (none seen in 20 independent T1 s). EDR1 is a member of the large Raf-like MKKKs family [41] and potentially any of the 48 members of this family (alone or in combination) could serve as a stomatal scaffold. As testing the entire family in not technically feasible, we used stomatal lineage expression data [26] to identify two close homologues of EDR1 expressed at the FAMA stage, MAP3K $\delta 1$ (At1g11850) and MAP3K $\delta 5$ (At4g24480). MAP3K $\delta 1$ and MAP3K $\delta 5$ mutants, however, were indistinguishable from $\mathrm{WT}$ in their response to FAMA stage MKK $5^{\mathrm{DD}}$ expression $(0 / 20$ independent $\mathrm{T} 1 \mathrm{~s}$ for each). Thus, the molecular identity of the stomatal scaffold remains of great future interest.

\section{Conclusions}

MKK4/5 face the problem of being used in early stages to repress stomatal progression, but being required for physiological regulation in guard cells. At the FAMA stage, MKK4/5 must therefore be actively rerouted from their previous role inhibiting stomatal development to allow terminal differentiation of guard cells (Fig. 9). A negative scaffold acting late in the stomatal lineage to redirect MKK4/5 would provide an elegant solution to a complex signal integration problem.

\section{Methods}

\section{Plant material and growth conditions}

All transgenic lines were generated in the Col-0 background. Seeds were plated on half-strength MurashigeSkoog media containing 1\% agar-agar (Caisson Laboratories, North Logan, UT) and $100 \mu \mathrm{g} / \mathrm{ml}$ Kanamycin (Sigma-Aldrich) or $50 \mu \mathrm{g} / \mathrm{ml}$ Hygromycin B (Life Technologies) when appropriate. Seedlings were grown under a light intensity of $100 \mu \mathrm{mol}$ photons $\mathrm{m}^{-2} \mathrm{~s}^{-1}$ in a $16: 8$ photoperiod at $22 \pm 1{ }^{\circ} \mathrm{C}$. Analyses were performed in 15 day after germination (dag) cotyledons unless stated otherwise. The mpk6-3 allele was Salk_127507 [42]. Mutant alleles for EDR1 (Salk_127158), MAP3Kס1 (Salk_ 048985) and MAP3K $\delta 5$ (Salk_029929 and Salk_036615) were obtained from the ABRC.

\section{Multiple sequence alignment and structural analysis}

Selected mammalian kinases were aligned using Clustal Omega [43] and structural models are in Additional file 3: Figure S3B. MKK5 and MKK7 structural predictions were performed with I-Tasser [27], using MEK1 (1S9J) to assist the prediction. Models were explored with SwissPdbViewer v4.1 and fit with the Magic Fit button [44]. Structural features were extracted from models and overlaid on the primary sequence (Additional files 3, 4 and 7: Figures S3-S5).

\section{Construction of constitutively active MKK and synthetic chimeras}

Domain swap constructs were assembled by fusion PCR from DNA amplicons (blocks) generated with Phusion ${ }^{\circ}$ High-Fidelity DNA Polymerase following manufacturer's instructions (New England Biolabs, Ipswich, MA). To generate blocks, MKK $5^{\mathrm{DD}}$ and $\mathrm{MKK} 7^{\mathrm{ED}}$ cloned into pENTR without stop codons or other chimeras were used as templates [22]. Blocks were designed to contain attL1 and attL2 functional sequences from pENTR to ease the cloning procedure through the Gateway strategy (Additional file 8). For domain swaps assembled from two blocks, 5' blocks contained the M13 forward priming site and attL1 recombination site before the MKK sequence; and 3' blocks contained the MKK sequence followed by attL2 recombination site and M13 reverse priming site. To facilitate fusion of the blocks, reverse primers for $5^{\prime}$ blocks and forward primers for 3' blocks were designed as chimeras of the two blocks to be fused, containing at least 15 bases from each block, and were completely complementary to each other. PCR products were gel extracted using QIAquick Gel Extraction Kit (QIAGEN Inc., Valencia, CA) and 1:1 M ratio mix were used as templates on fusion $\mathrm{PCR}$ reactions using M13 forward and reverse primers. Domain swap constructs were gel purified and cloned into pJET 1.2 according to CloneJET PCR Cloning Kit instructions (Thermo Life Sciences, Pittsburgh, PA). For domain swap constructs assembled from 3 blocks, $5^{\prime}$ and $3^{\prime}$ were generated with the same strategy as above, while internal block was amplified with forward and reverse chimeric primers. As domain swaps became more elaborate, first domain swap constructs were used as templates for generating new blocks. Primers, templates and sequences for each domain swap are listed in Additional file 8: Table S1, A and B. 
To build constructs for expression under SPCH and FAMA promoters, $2.5-\mathrm{kb}$ fragments previously described $[36,45]$ were first adapted to the Multisite Gateway system. Promoters were shuttled from pENTR to pDONR P4 P1R (Life Technologies, Grand Island, NY) by PCR amplification using promoter shuttling primers (Additional file 8) followed by BP recombination performed under manufacturer's instructions. Promoters flanked by attL4 and attR1 recombination sites (pDONR-promoter) were used in Multisite recombination reactions with domain swap constructs in PJET and R4pGWB440 [destination vector carrying the Gateway cassette flanked by attR4 and attR2 recombination sites, in frame C-terminal fusion to enhanced YFP and kanamycin selection in plants [46]]. Recombination reactions were performed in a two-step protocol. First, $1 \mu \mathrm{l}$ of LR Clonase II was added to $4 \mu \mathrm{l}$ vector mix (containing $150 \mathrm{ng}$ of pDONR-promoter and $150 \mathrm{ng}$ pJET-domain swap construct) and incubated at $25{ }^{\circ} \mathrm{C}$ for $5 \mathrm{~h}$. Then, $150 \mathrm{ng}$ of R4pGWB440 in $4 \mu \mathrm{l}$ solution were added to the reactions along $1 \mu \mathrm{l}$ of LR Clonase II. Reactions were incubated for additional $16 \mathrm{~h}$ at $25^{\circ}$ $\mathrm{C}$ and then stopped after the addition of $1 \mu \mathrm{l}$ of Proteinase $\mathrm{K}$ and incubation for $10 \mathrm{~min}$ at $37{ }^{\circ} \mathrm{C}$. Constructs were confirmed by sequencing and introduced in Arabidopsis by Agrobacterium tumefaciens-mediated transformation.

Mutants edr1, map $3 k \delta 1$ and map $3 k \delta 5$ were transformed with $F A M A_{\text {pro: }}: M K K 5^{D D}$ in pHGY, previously used in [22]. MPK3 and MPK6 clones were provided by Jean Colcombet (INRA Versailles-Grignon, France) [33].

\section{Scoring phenotypes and data analysis}

Seedlings (15 dag) were fixed in 7:1 ethanol:acetic acid, and cleared in Hoyer's medium. Cotyledons were imaged by differential interference contrast microscopy on a Leica DM2500 microscope at $\times 20$ magnification $\left(0.320 \mathrm{~mm}^{-2}\right.$ field of view). One picture per independent transgenic seedling was taken from the distal tip of the cotyledons, within the vascular loop, on the abaxial epidermis. Phenotypes at the FAMA stage were as follows: (1) normal phenotype, only single stomata with tolerance for 1 stomatal cluster per field of view; (2) stomatal inhibition, no stomata present or inhibited precursors coexisted with normal stomata and appeared in at least two independent fields of view per sample; (3) large stomatal cluster, at least two stomatal clusters (4 or more stomata in contact) per field of view; (4) small stomatal cluster, clusters contained 2-3 stomata in contact. When a sample contained a mixed population of clusters, the presence of large clusters defined the classification for this category. $\mathrm{MKK} 7^{\mathrm{ED}}$, clusters were systematically bigger than any chimera and often were delayed in development; to confirm clustering, older epidermis (near apical hydathode or in older plants) was scored. SPCH stage phenotypes were quantified as (1) inhibited (no stomata per field of view) or (2) not inhibited ( 2 or more stomata per field of view). To enable us to score phenotypes in T1 seedlings that must be grown on antibiotic selection, we grew 35S: YFP lines with the same antibiotic resistance as the MKK variants under the same conditions and scored these as the equivalent of WT controls.

A binomial distribution for phenotypic data was assumed and the percentages of each phenotype were calculated with a confidence of $95 \%$. This analysis is similar to others done on data of comparable nature [47]. Linear regressions between in vitro and in vivo data; and between SPCH and FAMA data in Fig. 7 and Additional file 6: Figure S7 were done with Microsoft Excel. To cluster chimeras, hierarchical clustering was performed on phenotypic data at the FAMA stage using the function pvclust in the statistical software R. Percentages of each one of the four phenotypes -Inhibited, Normal, Small Clusters and Large Clusters- were converted to frequencies (e.g. dividing by 100). The distance matrix was obtained by calculating the dissimilarities between all chimeras in their four phenotypes with the Manhat$\tan$ method. Clustering was performed with the ward.d method and the number of bootstrap replications was 1000. To statistically determine how similar chimeras were within clusters, a Chi-squared test of independence was implemented to compare phenotype distributions. Frequencies were compared to YFP (inactive), MKK $5^{\mathrm{DD}}$, MKK $7^{\mathrm{ED}}$ (inhibition of stomatal formation and stomatal clustering) and N5-MKK7 ${ }^{\mathrm{ED}}$ (strong stomatal inhibition). Chimeras that were not statistically different are noted with the same number in Fig. 7b. Unnumbered chimeras were statistically different from the rest $(p<0.05)$.

To test the role of selected MAP3Ks as putative negative scaffolds of $M K K 5^{\mathrm{DD}}$-mediated signaling at the FAMA stage, T1 seeds for wild type (Col) and mutants edr1, map $3 k \delta 1$ and map $3 k \delta 5$ carrying $F A M A_{\text {pro: }}: M K K 5^{D D}$ were plated on agar-solidified half-strength MurashigueSkoog media in the presence of $50 \mu \mathrm{g} / \mathrm{ml}$ Hygromycin B, and screened for stomatal lineage inhibition using bright field microscopy.

\section{Confocal microscopy}

Confocal images were collected using a Leica SP5 confocal microscope with excitation/emission spectra of $514 / 520$ to 540 for YFP and 565/580 to 610 for propidium iodide counterstaining. Image (NIH) was used to build Z-stacks from confocal images. To improve resolution of cell outlines, layers were summed rather than averaged. Z-stacks were then split into single channels and only the channel for the cell outlines was conserved, transformed into a grey-scale image and colors were 
inverted. Bright and contrast were modified to improve image quality. For localization, color channels in Zstacks were maintained and images were cropped. Localization of MKK-YFP chimeras was investigated in stable (T2-T3) lines with the exception of chimeras that induced stomatal inhibition, where localization was determined in $\mathrm{T} 1 \mathrm{~s}$ on antibiotic selection.

\section{Kinase-inactive MPK3 and MPK6 phosphorylation}

In vitro kinase assays to assess the ability of MKK variants to phosphorylate either kinase inactive (KI) MPK3 or MPK6 were performed as described in [23]. KI-MPKs were used to avoid autophosphorylation of the substrates. Band intensity was detected and analyzed using Image $(\mathrm{NIH})$. Each reaction was performed in triplicate. The ratio of phosphorylated KI-MPK/unphosphorylated KI-MPK detected by p42/44 antibody (Cell Signaling, Cat. No. 9102) was used to estimate MKK activity. Because this study is focused on regions in MKK5 and MKK7 responsible for differential activity, it was critical to be able to compare them side-by-side. For this reason, each replicate of the kinase assay was performed on all samples using the same "master mix", run on the same gel and blotted and probed together. To quantify MKK activities, the background signal intensities of unphosphorylated KI-MPKs were first subtracted from all samples within experiments. Because different experiments showed different signal intensities, signals were normalized to MKK5DD's (thus, defined as $100 \%$ kinase activity) within each experiment. Values for each MKK were then averaged across experiments and standard errors were calculated. Samples that showed "ghost bands" were eliminated from the analysis (see red boxes in Additional file 2: Figure S2).

\section{Yeast two-hybrid assays}

Yeast two-hybrid assays was performed with the matchmaker Two-Hybrid System 3 (Clontech) using a modified set of plasmids compatible with Gateway technology and conditions specified by the manufacturer. MKKs and chimeras were cloned as DNA Binding Domain fusions and MPKs were cloned as Activation Domain fusions. Three independent yeast colonies were tested for each pairwise comparison at 1, 1:10 and 1:100 dilutions after incubation of 2,3 and 4 days in plates containing $1 \mathrm{mM}$ 3-amino-1/2/4-triazole. Experiments were repeated three times with yeast cultures at OD600 of 1, 2 and 4. Interactions were evaluated as positive if significant growth was detected in 1:100 dilution at day 3 .

\section{Accession numbers}

Arabidopsis Genome Initiative locus identifiers for the genes studied in this work are: SPCH, AT5G53210; FAMA, AT3G24140; MKK5, AT3G21220; MKK6,
AT5G56580; MKK7, AT1G18350; MPK3, AT3G45640; MPK6, AT2G43790; EDR1, AT1G08720; MAP3K 1 , AT1G11850; MAP3K85, AT4G24480.

\section{Additional files}

Additional file 1: Figure S1. Yeast two-hybrid assay with native and chimeric MKKs and MPK3/MPK6. Representative yeast two-hybrid assay between MKK chimeras and MPK3/6 at 3 days of growth on control (-LW) and interaction (-LWH); three patches are serial (10 fold) dilutions. (JPEG 607 kb)

Additional file 2: Figure S2. In vitro kinase assays of MKKs and chimeras using kinase inactive (KI) MPK3 and MPK6 as substrates. Phosphorylation assays were performed as described in methods. Samples were separated in SDS-PAGE, transferred to PVDF membranes and probed with anti-pERK antibody. Western blots were quantified and average of experiments was used as an estimation of in vitro activity. Each lane is labeled with the figure where the data is presented (grey bar marked Figures), the kinase used with a graphic representation and with its full name in bottom panel. Samples unassigned to any figure (labeled as "-" in Figure) were not presented in the main figures of this manuscript for the sake of brevity, but are included here to allow us to keep the blots intact. Red squares highlight samples that were not considered for quantification due to detection artifacts. (JPEG $565 \mathrm{~kb}$ )

Additional file 3: Figure S3. Multiple sequence alignment [43] of catalytic domains in MPK kinases from mouse, rat, human and Arabidopsis thaliana. A, Conserved subdomains (Subdom.) and consensus sequences (Consen.) are represented on top of the alignment and follow the same codes and convention for an alignment of 60 different kinases by Hanks and Hunter [28]. In the consensus line: uppercase letters, invariant residues; lowercase residues, nearly invariant residues; 0 , positions conserving nonpolar residues; *, positions conserving polar residues; + , positions conserving small residues with near neutral polarity. Mammalian representative kinases selected for the alignment have been crystalized and belong to AGC group [CAMP-dependent kinase, cGMP-dependent kinase, etc.], CAMK group [Calcium-calmoduline-dependent protein kinase], CMGC group [cyclindependent kinase, mitogen-activated kinase, glycogen synthase kinase and cyclin-dependent-like kinase] and STE group [homologues of STE11 and STE20]. Gray boxes show CMGC insert [30] in ERK2, CDK2 and p38, and Pro-rich sequence (PRS, involved in binding the scaffold MP1) in MEK1 and MEK2 were included in the alignment and cause an expansion of subdomain X. Secondary structure $\left(2^{\circ} \mathbf{s t r}\right.$.) information is overlaid in the alignment (red for $a$-helices and yellow for $\beta$-strands). Conserved $a$-helices and $\beta$-strands are labeled following convention $[28,31]$. Due to CMGC insert and PRS, aG helix is located in two different regions of the alignment and was named differently (residues underlined): aG1 for MmPKA, HsARK-1, RnCaMKI, RnERK2, HsCDK2 and Hsp38; and aG2 for HsMEK1/2 and AtMKK5/7. In green text, missing residues in crystal structures from HsMEK1 and HsMEK2 which include the PRS. B, Table provides general information and structural model names for kinases used in this comparison. C, Multiple sequence alignment of $\mathrm{N}$ - and C-termini from human MEK1/2 and Arabidopsis MKK5/7. Sequences highlighted in gray correspond to first and last three amino acids of the catalytic domains shown in A. (JPEG $1120 \mathrm{~kb}$ )

Additional file 4: Figure S4. Multiple sequence alignment of C-termini in Arabidopsis and human MKKs. Top panel, partial sequences for all Arabidopsis and human MKKs were aligned with ClustalOmega. Loops $A$ and $B$ defined for Arabidopsis MKKs are highlighted in gray. Number of first amino acid of the partial sequence is noted to the left of each sequence. The long C-terminal extension of AtMKK3 is truncated in this figure. Bottom panel, genes used in the alignment. Name of genes for Arabidopsis correspond to Arabidopsis Genome Initiative (AGI) codes and GeneCards (GC) for humans. NCBI Gl, National Center for Biotechnology Information protein sequence identifier. (JPEG $1010 \mathrm{~kb}$ )

Additional file 5: Figure S6. Representative phenotype and subcellular localization of transgenic seedlings expressing FAMAp:N7-MKK6 ${ }^{\mathrm{EE}}-\mathrm{C7}$. (JPEG $465 \mathrm{~kb}$ ) 
Additional file 6: Figure S7. In vitro kinase activity of MKKs does not correlate with their in vivo activity. Linear regressions for MPK3 or MPK6 activity versus SPCH stage (A) or FAMA stage (B) activities, with their formulas and $R^{2}$, are displayed in the figure. FAMA stage activity was calculated as the addition of phenotypes different than normal (Inhibited, Small and Large clusters in Table 1). (JPEG 505 kb)

Additional file 7: Figure S5. Multiple sequence alignment for MKK4, MKK5, MKK7 and MKK9. Domains described in [23] are highlighted in blue and green. (JPEG $779 \mathrm{~kb}$ )

Additional file 8: Oligonucleotides sequences used in this paper, cloning strategy and protein sequence of chimeras. (DOCX $39 \mathrm{~kb}$ )

\section{Abbreviations}

CA-MKK: Constitutively active MKK; CDR: C-terminal distant region; CMGC: Cyclin-dependent kinases, MAPK, Glycogen synthase kinase and Cyclin-dependent kinase-like kinase; CPR: C-terminal proximal region; EDR1: Enhanced disease resistance 1; GC: Guard cell; GMC: Guard mother cell; KSR: Kinase Suppressor of Ras; MAPK/MPK: Mitogen-activated protein kinases; MEK: Mapk/Erk kinases; MKK: Mitogen-activated protein kinase kinase; MKK5 $^{\mathrm{DD}}$ : Constitutively active MKK5, MKK5 ${ }^{\mathrm{T215D}, \text { S221D; }}$ MKK $^{\mathrm{ED}}$ : Constitutively

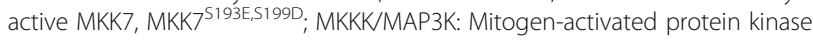
kinase kinase; MP1: Mek Partner 1; NtMEK2: Nicotiana tabacum MEK2; PRS: Proline-rich sequence; SPCH: SPEECHLESS; WT: Wild type; Y2H: Yeast two-hybrid; YFP: Yellow Fluorescent Protein

\section{Acknowledgements}

We thank Jean Colcombet (INRA Versailles-Grignon, France) for MPK3 and MPK6 clones. We also thank the current and past members of the Bergmann lab for their comments and insights into this study.

\section{Funding}

DCB is a Gordon and Betty Moore Foundation (GBMF) investigator of the Howard Hughes Medical Institute (HHMI). GBMF and HHMI did not participate in the design of the study and collection, analysis, and interpretation of data or in writing the manuscript.

\section{Availability of data and materials}

All the data supporting our findings is contained within the manuscript (Table 1 and Additional files 1, 2, 3, 4, 5, 6 and 7). Constructs are available upon request from DLW or DCB. Unprocessed micrographs available upon request from DLW. Protein and gene sequences were obtained from The Arabidopsis Information Resource (Arabidopsis.org) and the National Center for Biotechnology Information (NCBI) database.

\section{Authors' contributions}

DLW conceived this study, designed and performed in planta and yeast twohybrid experiments. GRL performed in vitro kinase assays and critically revised the manuscript. DLW and DCB wrote the manuscript. All materials generated in this manuscript are available upon request from DCB and DLW. All authors read and approved the final manuscript.

\section{Ethics approval and consent to participate}

Seeds were obtained from the Arabidopsis Biological Resource Center (ABRC), Ohio State University, Columbus, OH, USA. Experimental research was performed following Stanford University, state and federal guidelines for genetically modified organisms.

\section{Consent for publication}

$$
\text { Not applicable. }
$$

\section{Competing interests}

The authors declare that they have no competing interests.

\section{Publisher's Note}

Springer Nature remains neutral with regard to jurisdictional claims in published maps and institutional affiliations.

\section{Author details}

${ }^{1}$ Howard Hughes Medical Institute, Chevy Chase, USA. ${ }^{2}$ Instituto de Ingeniería Genética y Biología Molecular INGEBI - Consejo Nacional de Investigaciones Científicas y Técnicas, 1428 Buenos Aires, Argentina. ${ }^{3}$ Department of Biology, Stanford University, 371 Serra Mall, Stanford, CA 94305, USA

Received: 9 September 2017 Accepted: 28 March 2018

Published online: 10 April 2018

\section{References}

1. Keshet $Y$, Seger R. The MAP kinase signaling cascades: a system of hundreds of components regulates a diverse Array of physiological functions. In: Seger $R$, editor. MAP kinase signaling protocols. Second ed. Totowa: Humana Press; 2010. p. 3-38. Available from: https://doi.org/10.1007/978-1-60761-795-2_1.

2. Rodriguez MCS, Petersen M, Mundy J. Mitogen-activated protein kinase signaling in plants. Annu Rev Plant Biol. 2010;61:621-49. Available from: https://doi.org/10.1146/annurev-arplant-042809-112252.

3. Yang $\mathrm{SH}$, Sharrocks AD, Whitmarsh AJ. MAP kinase signalling cascades and transcriptional regulation. Gene. 2013;513:1-13. Elsevier B.V. Available from: https://doi.org/10.1016/j.gene.2012.10.033

4. Chen RE, Thorner J. Function and regulation in MAPK signaling pathways: lessons learned from the yeast Saccharomyces cerevisiae. Biochim Biophys Acta. 2007;1773:1311-40.

5. Dóczi R, Ökrész L, Romero AE, Paccanaro A, Bögre L. Exploring the evolutionary path of plant MAPK networks. Trends Plant Sci. 2012:17:518-25.

6. Andreasson $\mathrm{E}$, Ellis $\mathrm{B}$. Convergence and specificity in the Arabidopsis MAPK nexus. Trends Plant Sci. 2010;15:106-13. Available from: http://www. sciencedirect.com/science/article/pii/S1360138509002994.

7. Lampard GR, Macalister CA, Bergmann DC. Arabidopsis stomatal initiation is controlled by MAPK-mediated regulation of the bHLH SPEECHLESS. Science. 2008;322(5904):1113-6. https://doi.org/10.1126/science.1162263.

8. Bao MZ, Schwartz MA, Cantin GT, Yates JR, Madhani HD. Pheromonedependent destruction of the Tec1 transcription factor is required for MAP kinase signaling specificity in yeast. Cell. 2004;119:991-1000.

9. Brückner S, Köhler T, Braus GH, Heise B, Bolte M, Mösch H-U. Differential regulation of Tec1 by Fus3 and Kss1 confers signaling specificity in yeast development. Curr Genet. 2004;46:331-42. Available from: https://doi.org/10. 1007/s00294-004-0545-1.

10. Chou S, Huang L, Liu H. Fus3-regulated Tec1 degradation through SCFCdc4 determines MAPK signaling specificity during mating in yeast. Cell. 2004; 119:981-90.

11. Murphy LO, Smith S, Chen RH, Fingar DC, Blenis J. Molecular interpretation of ERK signal duration by immediate early gene products. Nat Cell Biol. 2002;4(8):556-64.

12. Murphy LO, Blenis J. MAPK signal specificity: the right place at the right time. Trends Biochem Sci. 2006;31(5):268-75.

13. Raman M, Chen W, Cobb MH. Differential regulation and properties of MAPKs. Oncogene. 2007;26:3100-12.

14. Good MC, Zalatan JG, Lim WA. Scaffold proteins: hubs for controlling the flow of cellular information. Science. 2011;332:680-6. Available from: http:// science.sciencemag.org/content/332/6030/680.

15. Schaeffer HJ, Catling AD, Eblen ST, Collier LS, Krauss A, Weber MJ. MP1: a MEK binding partner that enhances enzymatic activation of the MAP kinase Cascade. Science. 1998;281(5383):1668-71. http://science.sciencemag.org/ content/281/5383/1668.

16. Teis D, Wunderlich W, Huber LA. Localization of the MP1-MAPK scaffold complex to endosomes is mediated by p14 and required for signal transduction. Dev Cell. 2002:3:803-14.

17. McKay MM, Ritt DA, Morrison DK. Signaling dynamics of the KSR1 scaffold complex. Proc Natl Acad Sci U S A. 2009;106:11022-7. Available from: https://doi.org/10.1073/pnas.0901590106.

18. Zhang M, Wu H, Su J, Wang H, Zhu Q, Liu Y, et al. Maternal control of embryogenesis by MPK6 and its upstream MKK4/MKK5 in Arabidopsis. Plant J. 2017;92:1005-19. Available from: https://onlinelibrary.wiley.com/doi/abs/ 10.1111/tpj.13737. Cited 19 Dec 2017.

19. Jia W, Li B, Li S, Liang Y, Wu X, Ma M, et al. Mitogen-activated protein kinase cascade MKK7-MPK6 plays important roles in plant development and regulates shoot branching by phosphorylating PIN1 in Arabidopsis. PLoS Biol. 2016:14:e1002550. Estelle M, editor. Public Library of Science. Available from: https://doi.org/10.1371/journal.pbio.1002550. Cited 27 Sept 2016. 
20. Bergmann DC, Lukowitz W, Somerville CR. Stomatal development and pattern controlled by a MAPKK kinase. Science. 2004;304(5676):1494-7. http://science.sciencemag.org/content/304/5676/1494.

21. Wang H, Ngwenyama N, Liu Y, Walker JC, Zhang S. Stomatal development and patterning are regulated by environmentally responsive mitogenactivated protein kinases in Arabidopsis. Plant Cell. 2007;19(1):63-73.

22. Lampard GR, Lukowitz W, Ellis BE, Bergmann DC. Novel and expanded roles for MAPK signaling in Arabidopsis stomatal cell fate revealed by cell typespecific manipulations. Plant Cell. 2009;21(11):3506-17. https://doi.org/10. 1105/tpc.109.070110

23. Lampard GR, Wengier DL, Bergmann DC. Manipulation of mitogen-activated protein kinase kinase signaling in the Arabidopsis stomatal lineage reveals motifs that contribute to protein localization and signaling specificity. Plant Cell. 2014;26(8):3358-71. https://doi.org/10.1105/tpc.114.127415.

24. Won AP, Garbarino JE, Lim WA. Recruitment interactions can override catalytic interactions in determining the functional identity of a protein kinase. Proc Natl Acad Sci U S A. 2011;108:9809-14.

25. Popescu SC, Popescu GV, Bachan S, Zhang Z, Gerstein M, Snyder M, DineshKumar SP. MAPK target networks in Arabidopsis thaliana revealed using functional protein microarrays. Genes Dev. 2009;23(1):80-92. https://doi.org/ 10.1101/gad.1740009.

26. Adrian J, Chang J, Ballenger CE, Bargmann BOR, Alassimone J, Davies KA, et al. Transcriptome dynamics of the stomatal lineage: birth, amplification, and termination of a self-renewing population. Dev Cell. 2015;33:107-18. Available from: http://www.sciencedirect.com/science/article/pii/ S1534580715000684

27. Zhang Y. I-TASSER server for protein 3D structure prediction. BMC Bioinformatics. 2008;9:40. Available from: https://doi.org/10.1186/1471-2105-9-40

28. Hanks SK, Hunter T. Protein kinases 6. The eukaryotic protein kinase superfamily: kinase (catalytic) domain structure and classification. FASEB J. 1995:9:576-96. Available from: http://www.fasebj.org/doi/abs/10.1096/fasebj.9.8.7768349.

29. Kannan N, Neuwald AF. Evolutionary constraints associated with functional specificity of the CMGC protein kinases MAPK, CDK, GSK, SRPK, DYRK, and CK2alpha. Protein Sci. 2004;13(8):2059-77.

30. Goldsmith EJ, Akella R, Min X, Zhou T, Humphreys JM. Substrate and docking interactions in serine/threonine protein kinases. Chem Rev. 2007; 107:5065-81.

31. Knight JD, Qian B, Baker D, Kothary R. Conservation, variability and the modeling of active protein kinases. PLoS One. 2007;2(10):e982.

32. Brennan DF, Dar AC, Hertz NT, Chao WCH, Burlingame AL, Shokat KM, et al. A Raf-induced allosteric transition of KSR stimulates phosphorylation of MEK. Nature. 2011;472:366-9. Nature Publishing Group. Available from: https://doi. org/10.1038/nature09860

33. Berriri S, Garcia AV, Frei dit Frey N, Rozhon W, Pateyron S, Leonhardt N, et al. Constitutively active mitogen-activated protein kinase versions reveal functions of Arabidopsis MPK4 in pathogen defense signaling. Plant Cell. 2012;24:1-14

34. Ren D, Yang H, Zhang S. Cell death mediated by MAPK is associated with hydrogen peroxide production in Arabidopsis. J Biol Chem. 2002;277:559-65.

35. Li K, Yang F, Zhang G, Song S, Li Y, Ren D, et al. AlK1, a mitogen-activated protein kinase, modulates abscisic acid responses through the MKK5-MPK6 kinase cascade. Plant Physiol. 2017;173:1391-408. American Society of Plant Biologists. Available from: http://journals.plos.org/plosgenetics/article?id=10. 1371/journal.pgen.1004389.

36. Macalister CA, Ohashi-ito K, Bergmann DC. Transcription factor control of asymmetric cell divisions that establish the stomatal lineage. Nature. 2007; 445(7127):537-40.

37. Takekawa M, Tatebayashi K, Saito H. Conserved docking site is essential for activation of mammalian MAP kinase kinases by specific MAP kinase kinase kinases. Mol Cell. 2005;18:295-306.

38. Zhao C, Nie H, Shen Q, Zhang S, Lukowitz W, Tang D. EDR1 physically interacts with MKK4/MKK5 and negatively regulates a MAP kinase cascade to modulate plant innate immunity. PLoS Genet. 2014;10(5):e1004389. https://doi.org/10.1371/journal.pgen.1004389.

39. Cheng Z, Li J-F, Niu Y, Zhang X-C, Woody OZ, Xiong Y, et al. Pathogensecreted proteases activate a novel plant immune pathway. Nature. 2015; 521:213-6. Nature Publishing Group, a division of Macmillan Publishers Limited. All Rights Reserved. Available from: https:/doi.org/10.1038/nature14243.

40. Zhang $Y$, Wang $P$, Shao $W$, Zhu J-K, Dong J. The $\{B A S L\}$ polarity protein controls a $\{$ MAPK\} signaling feedback loop in asymmetric cell division. Dev Cell. 2015:33:136-49. Available from: http://www sciencedirect.com/science/ article/pii/S1534580715001409.
41. Ichimura K, Shinozaki K, Tena G, Sheen J, Henry Y, Champion A, et al. Mitogen-activated protein kinase cascades in plants: a new nomenclature. Trends Plant Sci. 2002;7:301-8. Available from: https://doi.org/10.1016/ S1360-1385(02)02302-6.

42. Müller J, Beck M, Mettbach U, Komis G, Hause G, Menzel D, et al. Arabidopsis MPK6 is involved in cell division plane control during early root development, and localizes to the pre-prophase band, phragmoplast, transGolgi network and plasma membrane. Plant J. 2010;61:234-48. Blackwell Publishing Ltd. Available from: https://doi.org/10.1111/j.1365-313X.2009. 04046.x.

43. Sievers F, Wilm A, Dineen D, Gibson TJ, Karplus K, Li W, Lopez R, McWilliam H, Remmert M, Söding J, Thompson JD, Higgins DG. Fast, scalable generation of high-quality protein multiple sequence alignments using Clustal Omega. Mol Syst Biol. 2011;7:539. https://doi.org/10.1038/msb.2011.75.

44. Guex N, Peitsch MC. SWISS-MODEL and the Swiss-Pdb Viewer: an environment for comparative protein modeling. Electrophoresis. 1997;18: 2714-23. Wiley Subscription Services, Inc., A Wiley Company. Available from: https://doi.org/10.1002/elps.1150181505.

45. Ohashi-ito K, Bergmann DC. Arabidopsis FAMA controls the final proliferation/differentiation switch during stomatal development. Plant Cell. 2006;18(10):2493-505.

46. Nakagawa T, Nakamura S, Tanaka K, Kawamukai M, Suzuki T, Nakamura K, et al. Development of R4 gateway binary vectors (R4pGWB) enabling highthroughput promoter swapping for plant research. Biosci Biotechnol Biochem. 2008;72:624-9. Available from: https://doi.org/10.1271/bbb.70678.

47. Wang W, Barnaby JY, Tada Y, Li H, Tör M, Caldelari D, et al. Timing of plant immune responses by a central circadian regulator. Nature. 2011;470:110-4.

\section{Submit your next manuscript to BioMed Central and we will help you at every step:}

- We accept pre-submission inquiries

- Our selector tool helps you to find the most relevant journal

- We provide round the clock customer support

- Convenient online submission

- Thorough peer review

- Inclusion in PubMed and all major indexing services

- Maximum visibility for your research

Submit your manuscript at www.biomedcentral.com/submit
) Biomed Central 